\title{
Properties and environment of radio-emitting galaxies in the VLA-zCOSMOS survey ${ }^{\star}$
}

S. Bardelli ${ }^{1}$, E. Schinnerer ${ }^{2}$, V. Smolčic ${ }^{3}$, G. Zamorani ${ }^{1}$, E. Zucca ${ }^{1}$, M. Mignoli ${ }^{1}$, C. Halliday ${ }^{4}$, K. Kovač ${ }^{5}$, P. Ciliegi ${ }^{1}$, K. Caputi ${ }^{5}$, A. M. Koekemoer ${ }^{6}$, A. Bongiorno ${ }^{7}$, M. Bondi ${ }^{8}$, M. Bolzonella ${ }^{1}$, D. Vergani ${ }^{1}$, L. Pozzetti ${ }^{1}$, C. M. Carollo ${ }^{5}$, T. Contini ${ }^{9}$, J.-P. Kneib ${ }^{10}$, O. Le Fèvre ${ }^{10}$, S. Lilly ${ }^{5}$, V. Mainieri ${ }^{11}$, A. Renzini ${ }^{12}$, M. Scodeggio ${ }^{13}$, G. Coppa ${ }^{1}$, O. Cucciati ${ }^{10}$, S. de la Torre ${ }^{14}$, L. de Ravel ${ }^{10}$, P. Franzetti ${ }^{13}$, B. Garilli ${ }^{13}$, A. Iovino ${ }^{14}$, P. Kampczyk ${ }^{5}$, C. Knobel ${ }^{5}$, F. Lamareille ${ }^{9}$, J.-F. Le Borgne ${ }^{9}$, V. Le Brun ${ }^{10}$, C. Maier ${ }^{5}$, R. Pellò ${ }^{9}$, Y. Peng ${ }^{5}$, E. Perez-Montero ${ }^{9,27}$, E. Ricciardelli ${ }^{12}$, J. D. Silverman ${ }^{5}$, M. Tanaka ${ }^{11}$, L. Tasca ${ }^{13}$, L. Tresse ${ }^{10}$, U. Abbas $^{15}$, D. Bottini1 ${ }^{13}$, A. Cappi ${ }^{1}$, P. Cassata ${ }^{16}$, A. Cimatti ${ }^{17}$, L. Guzzo ${ }^{14}$, A. Leauthaud ${ }^{18}$, D. Maccagni ${ }^{13}$, C. Marinoni ${ }^{19}$, H. J. McCracken ${ }^{20}$, P. Memeo ${ }^{13}$, B. Meneux ${ }^{7,28}$, P. Oesch ${ }^{5}$, C. Porciani ${ }^{21}$, R. Scaramella ${ }^{22}$, P. Capak ${ }^{23}$, D. Sanders ${ }^{24}$, N. Scoville ${ }^{3}$, Y. Taniguchi ${ }^{25}$, and K. Jahnke ${ }^{26}$

(Affiliations can be found after the references)

Received 1 July 2009 / Accepted 22 October 2009

\section{ABSTRACT}

Aims. We investigate the properties and the environment of radio sources with optical counterparts from the combined VLA-COSMOS and zCOSMOS samples. The advantage of this sample is the availability of optical spectroscopic informations, high quality redshifts, and accurate density determination.

Methods. By comparing the star formation rates estimated from the optical spectral energy distribution with those based on the radio luminosity, we divide the radio sources in to three families passive AGN, non-passive AGN, and star-forming galaxies. These families occupy specific regions of the 8.0-4.5 $\mu \mathrm{m}$ infrared color- specific star-formation plane, from which we extract the corresponding control samples.

Results. Only the passive AGN have a significantly different environmental distribution from their control sample. The fraction of radio-loud passive AGN increases from $\sim 2 \%$ in underdense regions to $\sim 15 \%$ for overdensities $(1+\delta)$ greater than 10 . This trend is also present as a function of richness of the groups hosting the radio sources. Passive AGN in overdensities tend to have higher radio luminosities than those in lower density environments. Since the black hole mass distribution is similar in both environments, we speculate that, for low radio luminosities, the radio emission is controlled (by fuel availability or confinement of the radio jet by local gas pressure) by the interstellar medium of the host galaxy, while in other cases it is determined by the structure (group or cluster) in which the galaxy resides.

Key words. galaxies: fundamental parameters - galaxies: general - galaxies: luminosity function, mass function - radio continuum: galaxies radio continuum: planetary systems

\section{Introduction}

Radio sources have been traditionally classified into two broad classes depending on their emission mechanism: AGN-driven emission has been proposed for early-type galaxies, while a starforming origin of the radio power has been invoked for late-type galaxies (Condon 1992). However, not all optical galaxies exhibit radio emission (at least at the depth of current surveys) and it is important to investigate the physical mechanism that triggers this emission. For AGN-induced activity, the difference between radio-quiet and radio-loud galaxies is naturally connected to the availability of fuel for the central engine, the black hole mass, and its emission efficiency (see e.g., Shabala et al. 2008, and references therein), while for star-forming objects it is closely related to the strength of the star-formation episode.

Heating by a central AGN is also thought to be important for the host galaxy evolution, providing the energy to stop the central cooling that drives gas to the central black hole in an

^ based on data obtained with the European Southern Observatory Very Large Telescope, Paranal, Chile, program 175.A-0839 and VLALarge Program VLA-AS0801. intermittent feedback mechanism dubbed "radio mode" (see Croton et al. 2006; Ciotti et al. 2009). The same intermittent mechanism is indirectly observed as bubbles in the intra-cluster medium (Birzan et al. 2004) or in the restarted activity of radio sources (Venturi et al. 2004; Bardelli et al. 2002). This heating emission is also proposed to be able to suppress star formation and to be an important mechanism for creating the galaxy color bimodality (Croton et al. 2006). Moreover, many radio AGN reside in early type galaxies (Ledlow \& Owen 1996), a class of objects that is preferentially found in high density environments.

It has been proposed that cluster/group mergers, galaxygalaxy mergings, or at least tidal disturbance caused by close encounters between galaxies should increase radio activity. For early-type galaxies, these phenomena could drive gas more efficently toward the center, while for late-type galaxies, these encounters should trigger and/or enhance star formation (see e.g. Bekki 1999; Vollmer et al. 2001). The radio luminosity of the AGN also depends on the interaction of the jet originating from the black hole with the surrounding gas of the host galaxy and/or of the hosting cluster (see e.g. Shabala et al. 2008, and references therein). For these reasons, some degree of dependence of radio 
activity on the environment is expected. Although the problem is clear from the qualitative description, controversial results are present in the literature and for mainly two reasons.

First of all, very few papers are primarly based on radio data. Usually, papers in the literature consider AGN selected on the basis of their optical (mostly estimated from line diagnostics) or X-ray activity (see Kauffmann et al. 2004, and references therein). It seems that the family of optical/X-ray AGN inhabits similar environments to non-active galaxies, with a preference for lower densities at higher stellar masses (Kauffmann et al. 2004; Silverman et al. 2009b). However, Best et al. (2005) claim that the phenomena generating the emission lines and the radio emission are statistically independent, even if recent results indicate that radio-loud emission-line AGN favour higher densities than radio-quiet ones (Kauffmann et al. 2008) (see also Caputi et al. 2009, for a study in the IR). Secondly, only specific environments have been considered, mainly clusters or groups (Miller et al. 2002; Hill \& Lilly 1991; Giacintucci et al. 2004), and therefore we are lacked coverage of a large dynamical range of densities.

The first paper that studied in a complete statistical way, both from the radio and optical side, the environment of radio galaxies was that of Best (2004). This paper uses data from the 2dF Galaxy Redshift Survey (Colless et al. 2001) and the NRAO VLA Sky Survey (Condon et al. 1998) with a clearly defined estimator of density, i.e., the projected density of the 10th nearest neighbor. Moreover, groups (and clusters) are found with the standard friend-of-friends detection method. The redshift range is $0.02<z<0.1$. The general result is that radio-selected star forming galaxies decrease in number with increasing overdensity, while objects with AGN activity exhibit little dependence on the local density, except at the very low densities, where the probability of finding these objects decreases significantly. Best (2004) also claims that the larger scale (i.e., on the scales typical of groups and clusters) is more important than the smaller scale (typical of galaxy pairs or companions) in determining the AGN radio emission. Star-forming galaxies do not exhibit any dependence on environment on scales larger than one Mpc (Kauffmann et al. 2004).

In this paper, we investigate the environmental properties of the radio sources present in the zCOSMOS survey using a set of well defined density estimators in the redshift range $0.1-0.9$. In Sect. 2 we present the data used and the estimators for various environments; in Sect. 3, we describe the method adopted to separate galaxies with radio emission induced by AGN from that induced by star formation and the definition of the control samples. In Sect. 4, we discuss the differences between the various samples, while in Sect. 5, we present the dependences on environment. In Sect. 6, the luminosity distributions and radiooptical ratio for a specific class of radio AGN are shown and in Sect. 7, we discuss our results. The adopted cosmology has $\Omega_{\mathrm{m}}=0.25, \Omega_{\Lambda}=0.75$, and $H_{0}=70 \mathrm{~km} \mathrm{~s}^{-1} \mathrm{Mpc}^{-1}$.

Throughout the paper we adopt the term "radio galaxy" for galaxies hosting a radio source, independently of the physical nature of the emission.

\section{Data and density estimators}

To select the optical sample, we used the zCOSMOS Bright survey, a redshift survey with a magnitude selection of $I_{A B}<$ 22.5 (Lilly et al. 2007, 2009) that has been undertaken in the $1.7 \mathrm{deg}^{2}$ of the COSMOS field (Scoville et al. 2007), imaged by the Hubble Space Telescope / Advanced Camera for Surveys F814W I-band (Koekemoer et al. 2007).
The observations have been carried out with the 8-m ESO Very Large Telescope using VIMOS, a multi-slit spectrograph with a resolution of $R \sim 600$, resulting in an rms accuracy in the velocity determinations of $\sim 110 \mathrm{~km} \mathrm{~s}^{-1}$ (Lilly et al. 2007, 2009)

This paper is based on the first $\sim 10000$ spectra, which have a rather non-uniform sampling pattern (see Fig. 5 in Lilly et al. 2009) over an area of $1.40 \mathrm{deg}^{2}$ and an average sampling rate of $\sim 33 \%$. From this sample, we extracted a "statistical sample" of 8481 galaxies with high quality redshift determination (flag $>$ 1.5) and excluded broad-line AGN. Note that within our redshift limit of 0.9 adopted in the following analysis, the number of broad-line AGN is 17, of which 3 are radio loud.

Absolute magnitudes were computed following the method of Ilbert et al. (2005) from the COSMOS photometry (see Zucca et al. 2009), which covers a wide range of wavelengths from the UV to the NIR. This method computes the rest-frame absolute magnitudes using the nearest observed band (at the redshift of the galaxy) plus a correction derived from a template fit, and is the least template-dependent method possible.

We used stellar masses $\left(M_{\text {star }}\right)$ and star formation rates (SFRs) computed by Bolzonella et al. (2010) using Hyperzmass (see details in Bolzonella et al. 2010; Pozzetti et al. 2007) by means of a spectral energy distribution fit to the photometric data. The method uses the Bruzual \& Charlot (2003) library and the Chabrier (2003) initial mass function. The library provides a model of a simple stellar population and its evolution in 220 age steps. From this database, Hyperzmass imposes a set of $10 \mathrm{ex}-$ ponentially decreasing star formation histories (with $e$-folding times ranging from 0.1 to $30 \mathrm{Gyr}$ plus a model of continuous star formation) to fit the photometric data. We assume the values of stellar mass and star formation rate to be those corresponding to the best-fit template. The typical statistical error in the stellar masses is estimated to be $\sim 0.20$ dex.

The usually adopted conversions between radio luminosity and star formation rate are based on the Salpeter (1955) initial mass function and for this reason we corrected our $\log M_{\text {star }}$ and $\log (S F R)$ by adding 0.23 and 0.19 , respectively, which are the median differences of the two quantities computed with the two initial mass functions.

For the radio band, we used the VLA-COSMOS survey, a large project consisting of 23 pointings in an area of $1.4 \times$ 1.4 square degrees (Schinnerer et al. 2007; Bondi et al. 2008). The resolution is $1.5 \times 1.4 \operatorname{arcsec}^{2}$ and the survey, which has a mean rms of $\sim 10.5 \mu \mathrm{Jy}$, detected 2501 sources at more than $5 \sigma$.

Ciliegi et al. (in prep.) correlated this sample with the COSMOS optical samples (Capak et al. 2007), finding 2060 radio sources with optical counterparts. We correlate the zCOSMOS "statistical sample" with the VLA-COSMOS sample obtaining a final radio sample of 315 radio galaxies observed spectroscopically out of the 1231 radio sources with $I_{A B}<22.5$. We note that our radio data sampling rate is slightly lower than the average zCOSMOS sampling rate because the zCOSMOS survey does not cover the entire VLA-COSMOS area.

Radio luminosities were computed assuming that the radio spectrum is a power law function (defined by $S \propto v^{\alpha}$ ) with a slope $\alpha=-0.7$.

We used the density estimtes computed by Kovač et al. (2010) using the ZADE algorithm, which maximizes the statistical robustness of the estimators using both the spectroscopic and the photometric redshift samples. The algorithm offers the densities computed for each galaxy both as a function of the distance of the $n$ th-nearest neighbour and in spheres of fixed comoving radius. These densities are also computed by weighting the galaxies by either their $B$ band luminosities or stellar masses. 
As tracers of the density field, galaxies both of a flux-limited and volume-limited sample were used.

The method was tested on mock cosmological simulations, by extracting samples with the same observational limits as zCOSMOS. The overdensities were found to be correctly estimated at all redshifts with a slight $(\sim 20 \%)$ underestimate of the true value at low densities, corresponding to $(1+\delta)<0.1$ (where $\delta=\rho-\langle\rho\rangle /\langle\rho\rangle)$. In the following, we use the densities derived with the nearest neighbor approach.

In addition, the zCOSMOS optically-selected group cata$\log$ (Knobel et al. 2009) offers a complementary perspective on the role of environment (Iovino et al. 2010). These groups were extracted using a combination of the friends-of-friends method with a Voronoi tesselation density estimator on the zCOSMOS spectroscopic catalogue. The method, applied to the statistical sample, detected $\sim 800$ groups, 103 of which have more than 5 members. The fraction of galaxies in groups is $\sim 20 \%$ with some dependence on redshift. Estimates of the group richness were calculated using the number of galaxies of the volume-limited sample, considered as proxies of the total richness of groups.

\section{AGN versus star forming galaxies in environmental studies}

There are essentially two steps in studying environmental effects for radio sources: the division of radio sources according to AGN and star-forming-induced radio emission and the definition of coherent control samples. In fact, it is already known that radio AGN reside mostly in early-type galaxies and therefore their overdensity distribution has to be compared only with that of radio-quiet early-type galaxies, otherwise any possible difference observed in environment could just be due to the optical morphology-density relation (Dressler 1980).

However, a sharp division between AGN and SF galaxies in some cases has little physical meaning, because we know that both phenomena are commonplace in a number of galaxies (see e.g. Silverman et al. 2009a) and it is therefore difficult to determine the fraction of the radio flux that originates from AGN and/or from the star formation activity.

For AGN/SF galaxy luminosity function studies (e.g. Bardelli et al. 2009; Smolčic et al. 2009a,b), the aim is to count the total number of AGN (or star-forming galaxies) and for these studies it is correct to consider all objects exhibiting AGN (SF) activity, regardless of its relative contribution to the radio flux. For density studies, the situation is slightly different. In this case, we ask whether the environment has (or not) any effect in triggering radio emission from $\mathrm{AGN}$ or star formation and it is not easy to reach a conclusion in those composite objects, considering that the trigger mechanisms are likely very different.

In other words, if a class of radio sources exhibits an environmental dependence, is this related to its star-forming or to the AGN component of the flux?

In the following, we present a method for selecting subsamples in such a way that the "radio AGN sample" contains radio sources for which the AGN emission is at least about one order of magnitude higher than that expected from the star formation. Therefore, our definition of radio AGN is "an object for which the radio emission is largely dominated by the emission of the central engine".

With this definition, optical AGN defined on the basis of emission line ratios, are included in the star forming galaxy sample if they have a low ratio of AGN/SF radio flux. This inclusion is supported by a) the independence of emission line and radio emission (Best et al. 2005), at least for low luminosity AGN such as ours; b) the independence of these AGN from the environment (Silverman et al. 2009b), similar to what was a posteriori found for star-forming galaxies; and c) the number of optical AGN with radio emission, which is negligible with respect to the number of star-forming galaxies.

\subsection{AGN versus star-formation radio emission}

There are several recipes for dividing the radio population into sources with emission from an AGN or from star formation (SF) (see e.g., Smolčic et al. 2008; Best et al. 2005, and references therein). They are mainly based on determining the optical properties of known radio-emitting star-forming galaxies and defining a limit to the emission of the AGN. In particular, the method of Best et al. (2005) separates the two classes on the basis of the $D_{n}(4000)-L_{1.4 \mathrm{GHz}} / M_{\text {star }}$ plane. In practice, this method compares the ages of stars (estimated by the $D_{n}(4000)$ parameter) with the current specific star formation rate (estimated with $\left.L_{1.4 \mathrm{GHz}} / M_{\text {star }}\right)$ and the chosen separation line is 0.225 above the $D_{n}(4000)$ value of a 3 Gyr exponentially decaying star-formation galaxy track. The method of Smolčic et al. (2008) is based on the two families defining two peaks in a PCA-based rest-frame colors combination. Both methods were calibrated with the use of line diagnostics.

Our method instead has two steps. First, we compare the star formation rates estimated from the radio emission and the spectral energy distribution fit (Bolzonella et al. 2010) and we separate AGN from SF radio sources on the basis of this comparison. For the estimate of the star formation rate from the radio emission, we used the Bell (2003) formula. Second, we study the optical and infrared properties of the radio galaxies to define control samples with similar properties.

We note that our results do not depend strongly on the specific formula adopted in converting $L_{\text {radio }}$ to SFR because our method is based on a relative comparison, which considers as AGN all objects outside a given radio-optical star formation correlation. For the zCOSMOS spectra, we could also use the star formation rates derived from the lines, but we decided to use the SED determinations because: a) at low redshifts $(z<0.5)$, the emission-line estimate would use the $\mathrm{H} \alpha$ line (which is affected by problems with sky line contamination and strong fringing in the spectra in the redshift range [0.3-0.5]), while at higher redshift the usable line is [OII] and b) for weak star formation, the line measurements are able to provide only upper limits. We note that because of point a), the SFR estimate would not be homogeneous being based on two different lines in two different redshift ranges. Moreover, by considering only detected lines, the final usable sample would be $\sim 60 \%$ of that we have with SED determinations. For this reason, we only use the spectral informations only to calibrate the method.

In Fig. 1 (upper panel), we show the SFR(radio) $S F R$ (SED) plane for the entire VLA-COSMOS sample limited to $I<22.5$ ) and its zCOSMOS subsample (red points). Only in this case, used for illustratory purpose, we used the photometric redshifts as derived by Ilbert et al. (2009) both for computing distances and SEDs.

It is clear that at high $\left(>10 M_{\odot} \mathrm{yr}^{-1}\right)$ values of $S F R(\mathrm{SED})$, the two quantities are reasonably correlated, defining a "starforming galaxy line", while at low values (SFR(SED) < $\left.2 M_{\odot} \mathrm{yr}^{-1}\right)$ a significant population of radio sources with a radio star formation "excess" is present. This excess means that 
the radio emission of this population is not driven by star formation and thus is due to AGN.

However, we need to test the robustness of the SED star formation rate determination. By taking advantage of having the zCOSMOS spectra, we can apply standard line diagnostics (Baldwin et al. 1981) to classify emission-line galaxies as star forming, Seyfert 2, or LINERS. This procedure was applied in an automated way to the zCOSMOS sample (Bongiorno et al. 2010) following the method of Lamareille et al. (2009). At low redshifts $(0.15<z<0.45)$ the $[\mathrm{OIII}] / \mathrm{H} \beta$ versus $[\mathrm{NII}] / \mathrm{H} \alpha$ ratios have been used (red diagnostics), while at higher redshift $(0.5<z<0.93)$ the objects have been classified using the line ratios $[\mathrm{OIII}] / \mathrm{H} \beta$ versus $[\mathrm{OII}] / \mathrm{H} \beta$ (blue diagnostics).

From the 133 radio detected galaxies classified as star forming, we selected a subsample of 83 "bona fide star-forming galaxies", i.e., classified as SF galaxies from diagnostics and with high quality line measurements and emission lines far away from sky and fringing lines. In Fig. 1 (lower panel), we plot the ratios of the SED (black points) or line (red and blue points) SFR to the radio SFR determination versus the radio SFR. Red points correspond to SFR obtained from the [OII] line and blue points from the $\mathrm{H} \alpha$ line (Moustakas et al. 2005). Note that SED and emission line determined SFR are consistent with each other, but their ratios with the radio SFR shows a significant decrease with increasing SFR(radio).

In particular, assuming that the values of the radio SFR represent the true ones, the SFRs derived from both the SED and emission lines overestimate the low and underestimate the high SFR. We note that the underestimate of the SFR from the $\mathrm{H} \alpha$ line (red points) for $S F R>1 M_{\odot} \mathrm{yr}^{-1}$ is qualitatively similar to the result of Caputi et al. (2008), found by comparing this estimation with the value derived from the combined UV and IR luminosity (see their Fig. 8).

We fitted the points in the figure (the "bona fide star-forming galaxies") with a linear relation finding that $\langle\log (S F R[S E D])\rangle=$ $0.55 \log (S F R[$ Radio $])+0.347$, where $\langle\log (S F R[$ SED $])\rangle$ is the mean value of $\log (\mathrm{SFR}[\mathrm{SED}])$ at a given $\log (S F R$ [Radio]). We then computed the "corrected" $S F R[S E D]_{c}$ by applying the relation

$$
\begin{aligned}
\log \left(S F R[\mathrm{SED}]_{\mathrm{c}}\right)= & \log (\operatorname{SFR}[\mathrm{SED}])-\langle\log (\operatorname{SFR}[\mathrm{SED}])\rangle \\
& +\log (\text { SFR }[\text { Radio }]) .
\end{aligned}
$$

In practice, we force the two estimates of SFR for the "bona fide star-forming galaxies" to statistically follow the relation $\log \left(S F R[\mathrm{SED}]_{\mathrm{c}}\right)=\log (\operatorname{SFR}[$ Radio $])$. The rms dispersion around the fit is $\sim 0.35$.

Finally, we define the division line between SF galaxies and $\mathrm{AGN}$ to be the line parallel to the $\log \left(S F R[\mathrm{SED}]_{\mathrm{c}}\right)=$ $\log (S F R[$ Radio] $)$ relation and shifted horizontally by 3 standard deviations. In this way, to be included in our AGN sample, the radio emission from the black hole should be about an order of magnitude higher than the average emission due to star formation.

Obviously, this division line between star formation and AGN is somewhat subjective, because it is likely that there is also some AGN contribution to the radio flux also in objects below the division line. However, this procedure ensures that we define a "clean", even if not complete, AGN sample. In particular, at higher star formations rates, we cut at progressively higher radio luminosities.

Applying this procedure, we define a sample of 97 AGN out of our total sample of 315 radio sources (Fig. 2).

Among the 133 automatically identified star-forming galaxies, only 13 are in the AGN region. After visual checks of the
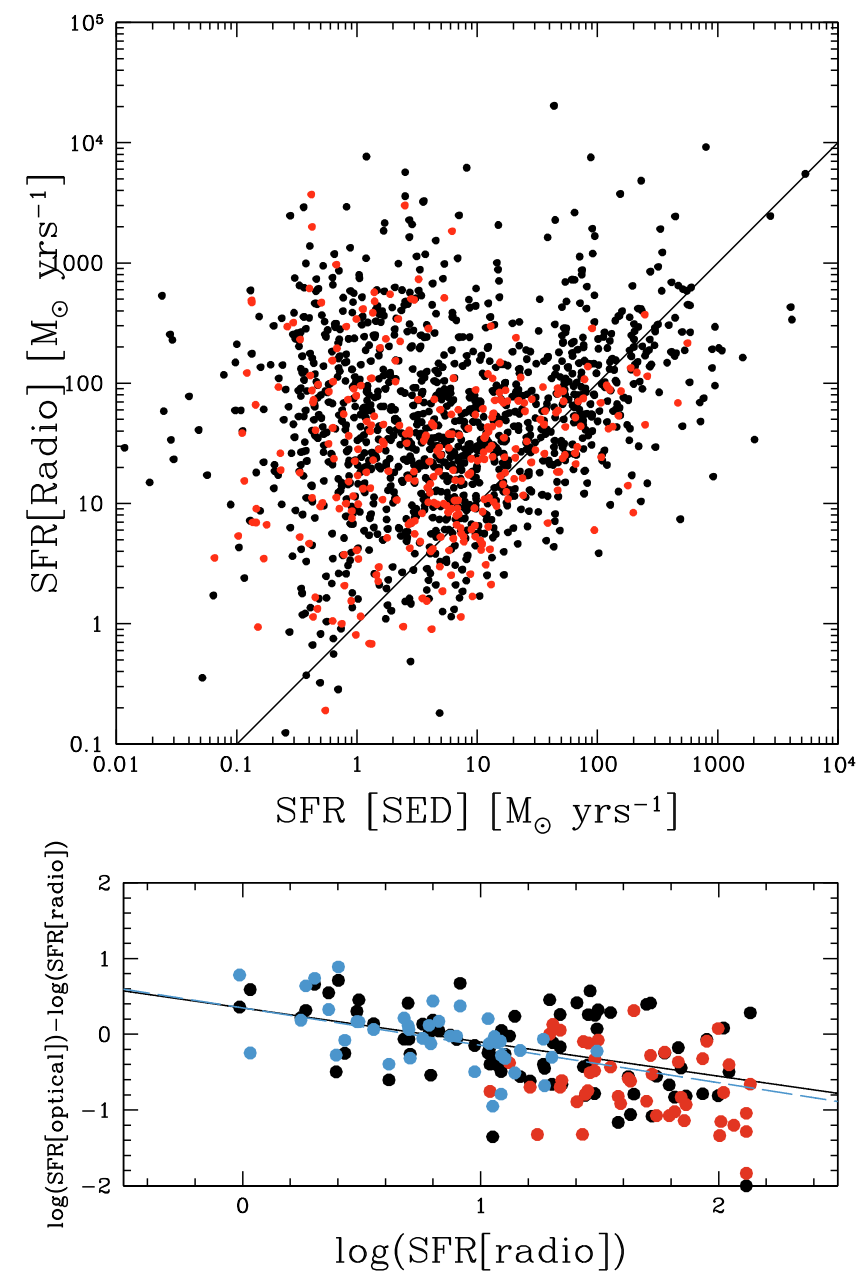

Fig. 1. Upper panel: SFR(radio) versus $\mathrm{SFR}(\mathrm{SED})$ for all radio galaxies in the initial VLA-COSMOS sample with $I<22.5$ (black circles). In red, the 315 objects within the zCOSMOS survey are highlighted. The solid, black line is the $S F R(\mathrm{SED})=S F R$ (radio) line drawn for reference. Lower panel: Ratio of the SFR as determined in the optical (from SED and lines) and the SFR estimated from radio luminosity for a sample of "bona fide" star-forming galaxies (see text). In red, the SFRs obtained from the [OII] line, while in blue that obtained from the $\mathrm{H} \alpha$ line. Black points refer to SFRs determined from SED. Blue and black lines are the least squares fits to line and SED determination.

spectra, we conclude that all but two could be considered incorrect or at least uncertain classifications because of the low signalto-noise of the lines or contamination by the sky/fringing lines. This shows that a large fraction $(>90 \%)$ of the objects classified as star-forming galaxies on the basis of their optical spectral diagnostics are indeed recognized as such by our method.

As expected, the situation for optically classified AGN is less clear: among the 14 LINERs and 11 Seyfert 2 objects, the galaxies showing "star-formation excess" are 2 and 3, respectively. Assuming that for our optical AGN the average contribution of the AGN to the line luminosity is $\sim 50 \%$ (see Fig. 6 of Silverman et al. 2009a) and comparing the expected radio star formation from the optical-radio star formation correlation with the observed radio luminosity, we conclude that, on average, the AGN radio luminosity of these objects is a factor $\sim 3-4$ higher than that due to star formation.

Note that although the $S F R$ (optical) - SFR(radio) plot is useful for distinguishing AGN from star-forming galaxies, it cannot be used to extract a consistent control sample, because the 


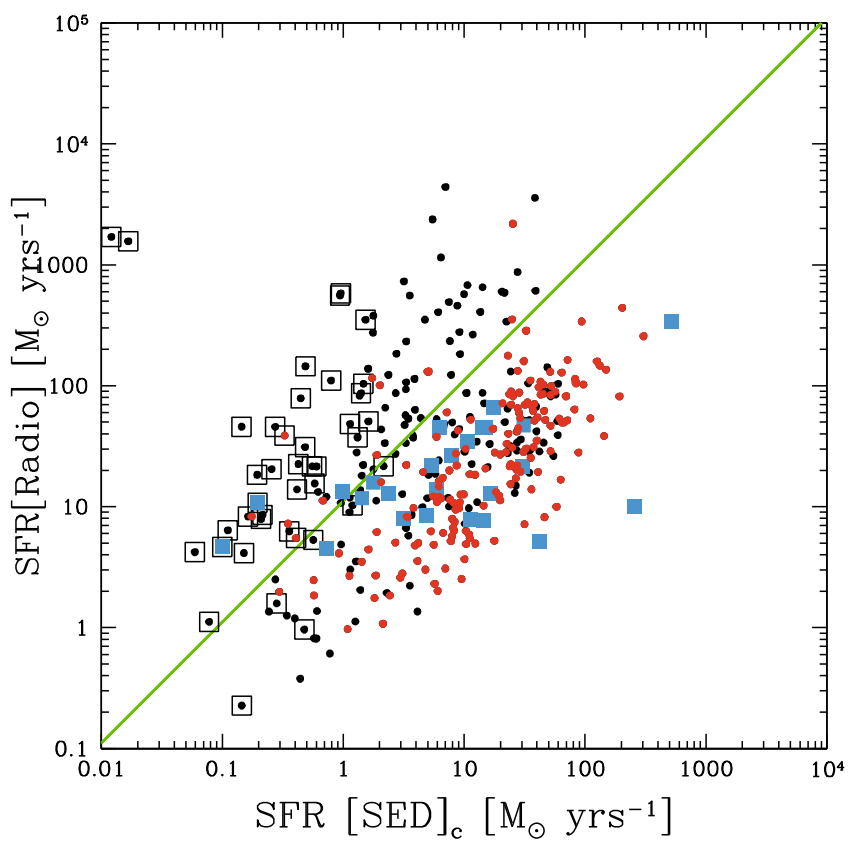

Fig. 2. SFR(radio) versus the corrected (see text) SFR(optical) for our zCOSMOS sample. Red points are star forming galaxies, blue squares are optical AGN (LINERs and Seyfert 2) as determined by line diagnostics, while empty squares are radio emitting objects in the passive region in the infrared colors-specific star formation plane (see Sect. 3.2). The green line is the adopted division between star-forming galaxies and AGN. Small black points are objects without spectral diagnostic classification (due to low quality line measurements).

division between galaxy classes is not derived using optical properties only.

\subsection{The color-specific star-formation plane}

We now discuss the relation between the infrared color 8.0-4.5 $\mu$ m obtained by the Spitzer satellite (Sanders et al. 2007) and the optical specific star formation rate (SSFR). It is already known that near infrared colors provide a powerful method in finding broad line AGN (see e.g., Lacy et al. 2004), but here we are more interested in the behavior of the "normal" galaxy population. We define the infrared color as the ratio of the two fluxes $S_{8.0} / S_{4.5}$.

In the upper panel of Fig. 3 we show the distribution of the galaxies of the statistical sample (as defined in Sect. 2), where the star-forming galaxies are indicated in red, the Seyfert 2 galaxies in green, and the LINER galaxies in blue, all classified with spectroscopic diagnostics. we note a number of nonclassified objects (black points) that reside in the star forming galaxies, where the emission lines are expected to be strong. These classification failures can be explained by noting that the redshift distribution of these objects has two peaks at $z \sim 0.5$ and $z \sim 0.85$. The first peak, corresponds to the point in which the $\mathrm{H} \alpha$ line is at the red border of the observed spectrum and affected by fringing, and the [OII] line begins to enter from the blue border. At the high redshift peak, the $\mathrm{H} \beta$ emission-line starts to be affected by fringing.

The existence of two populations is rather obvious and is related to the bimodality also found in the optical by using $B-I$ or $U-B$ colors (see Cucciati et al. 2010) to divide red, passive galaxies from star-forming ones.
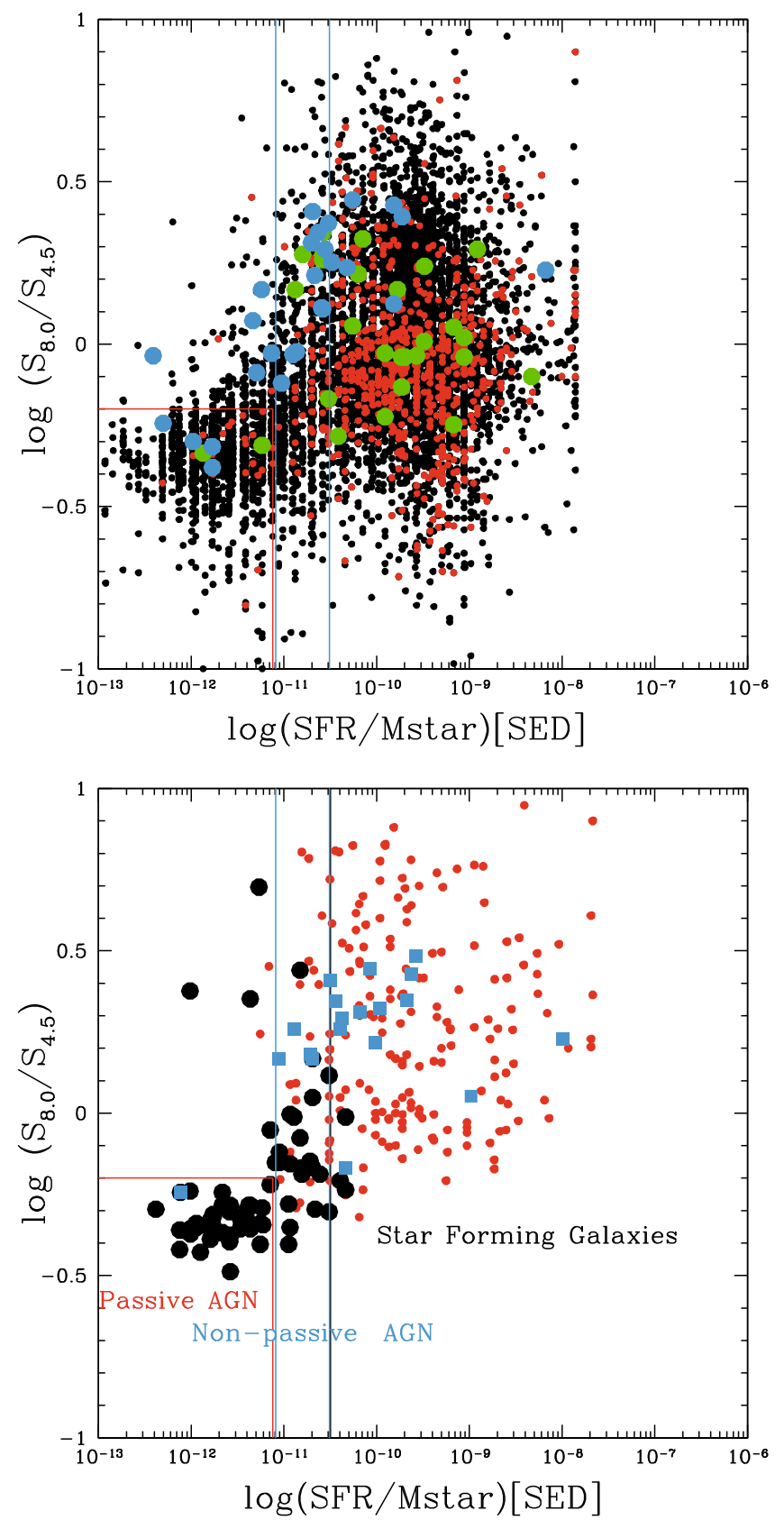

Fig. 3. Upper panel: infrared color versus specific star-formation rate for all galaxies in the statistical sample. Red points represent the star forming galaxies, blue points the LINERS and green points the Seyfert 2 objects classified by line diagnostics. Black points represent unclassified galaxies (see text). Lower panel: same as the upper panel, showing only the radio-galaxies. The large circles represent our defined radio AGN. Blue squares are the optical AGN (not divided into LINERs and Seyfert 2). Vertical and horizontal lines represent the division between the three galaxy populations defined in the text.

Interestingly, Seyfert 2 objects occupy the same locus of starforming galaxies, while LINERs are at the upper border (see also Fig. 19 of Smolčic et al. 2008).

Passive galaxies are located in a clump at the lower left of the plot, while the star-forming galaxy region has a vertex close to the passive clump that opens at greater distance. The division value was chosen to be the value of SSFR which show a minimum in the density distribution of the galaxies. 
Table 1. Sample definition: numbers in the third and fourth columns are with and without the "evolving" magnitude limit.

\begin{tabular}{llll}
\hline \hline Sample & Limits & Number in radio sample & Number in control sample \\
\hline "passive AGN" & $\log \left(S F R / M_{\text {star }}\right)[\mathrm{SED}]<-11.1$ and $\log \left(S_{8.0} / S_{4.5}\right)<-0.2$ & $38 / 38$ & $524 / 530$ \\
"non-passive AGN" & $-11.1<\log \left(S F R / M_{\text {star }}\right)[\mathrm{SED}]<-10.5$ & $31 / 37$ & $496 / 581$ \\
star forming galaxies & $\log \left(S F R / M_{\text {star }}\right)[\mathrm{SED}]>-10.5$ & $154 / 186$ & $1672 / 3154$ \\
\hline
\end{tabular}

In the lower panel of Fig. 3, we show the same plot but for radio sources alone. The large, black circles indicate the radio AGN objects defined in the previous subsection. This plot shows that the radio AGN sample consists of two different populations, one corresponding to the passive galaxies $\left(\log \left(S_{8.0} / S_{4.5}\right)<-0.2\right.$ and $\left.\log \left(S F R / M_{\text {star }}\right)<-11.1\right)$ and one extending toward the SF galaxies locus.

It also appears as if there is a specific star formation limit (at $\left.\log \left(S F R / M_{\text {star }}\right) \sim-10.5\right)$, at which radio AGN are rare when SSFR is higher than this limit. The division at $\log \left(S F R / M_{\text {star }}\right) \sim$ -10.5 is certainly subjective and may depend on the characteristics of the survey, but it is a fact that the majority of radio AGN are found on the left of the line and not spread throughout the plot. This division value was chosen to maximize the number of radio AGN and minimize the contamination from $\mathrm{SF}$ galaxies in the region defined for AGN.

Among the 75 AGN radio sources with measured infrared color, 38 are passive galaxies, while the remainig are in the second region defined above. We note that this behavior is similar to that shown in Fig. 10 of Smolčic et al. (2008) using the $8.0-4.5 \mu \mathrm{m}$ and 5.8-3.6 $\mu \mathrm{m}$ color plane.

In Fig. 2, a number of radio sources are defined as passive that are not included in the passive AGN sample, because they are not sufficiently radio luminous to selected as AGN. Assuming the extreme hypothesis that all of these objects are passive AGN, this would imply that the incompleteness of our sample is at the level of $\sim 15 \%$. Recomputing all quantities after including of these objects, the following results do not change.

As a general conclusion, we define as radio AGN all objects with "radio star formation excess", while "passive AGN" occupy the passive (i.e., very low specific star formation) galaxy peak of Fig. 3. The remaining AGN are defined as "non-passive AGN", while all other radio sources are considered to be star-forming galaxies.

As outlined above, this choice is justified in the light of the analysis of the environmental dependencies. It is worth emphasizing that a more sophisticated method to separate AGN and star-forming galaxies, when both phenomena are present, is still required. Our method produces a star-forming galaxy sample contaminated by AGN at a level of $~ 8 \%$ (after having applied the limits of Table 1). We checked that this contamination has no effect on the following conclusions.

Therefore, the control sample is extracted using the limits taken from Fig. 3 and reported in Table 1. The only exception is the additional cut that we applied to the stellar mass in the passive AGN sample, which is discussed in Sect. 4.2.

\subsection{Comparison with other classifications}

To check the consistency between our method for classifiying AGN and SF galaxies and other methods in the literature, we consider the spectroscopic line diagnostics (Baldwin et al. $1981)$, the $D_{n}(4000)-L_{1.4 \mathrm{GHz}} / M_{\text {star }}$ plane of Best et al. (2005), and the Smolčic et al. (2008) method. In Fig. 4, we plot the $[\mathrm{OIII}] / \mathrm{H} \beta$ versus $[\mathrm{NII}] / \mathrm{H} \alpha$ the equivalent width ratios (red diagnostics) and the $[\mathrm{OIII}] / \mathrm{H} \beta$ versus $[\mathrm{OII}] / \mathrm{H} \beta$ (blue diagnostics)
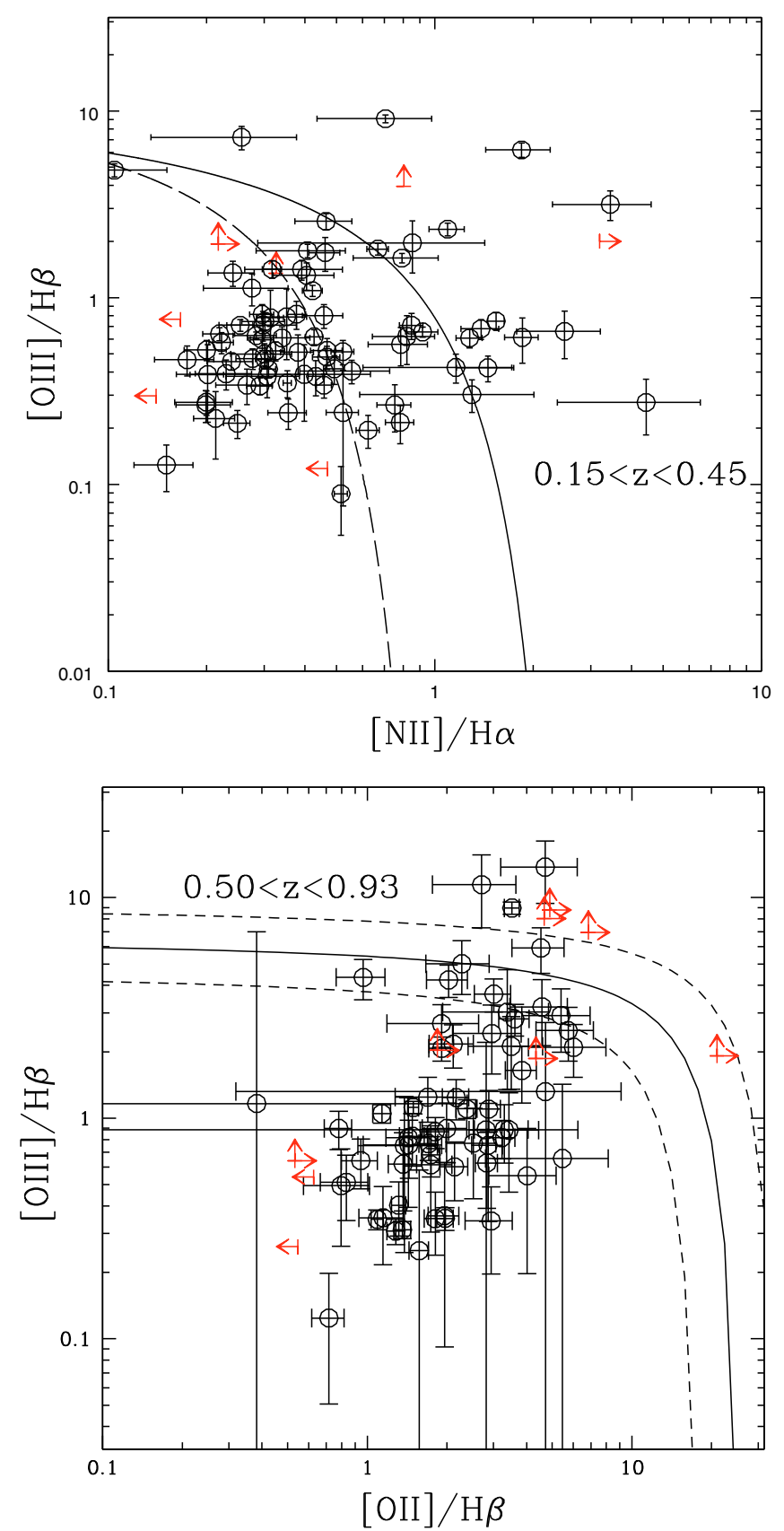

Fig. 4. Red and blue diagnostics diagram for our star-forming galaxies with radio detection. Circles are objects with all lines detected. For the upper or lower limits, we plot only objects with only one upper limit determination for each line ratio.

line diagnostics for radio SF galaxies as defined by our method. We recall that the two diagnostics were applied to two redshift ranges ([0.15-0.45] and [0.5-0.93], respectively). Circles are objects for which all emission lines was detected. Here, an upper or lower limit implies that for each line ratio one line has been 
detected and for the other we have an upper limit. When we have upper limits for both lines, no point is shown in the diagram.

In the upper panel, the solid line is the theoretical Kewley et al. (2001) division between SF galaxies and AGN, while the dashed line is the Kauffmann et al. (2003) line. The region between the Kewley et al. (2001) and the Kauffmann et al. (2003) lines defines a region of so-called "composite galaxies". In the lower panel, the division proposed by Lamareille et al. (2004) is plotted as a solid line, and the dashed lines represent the $+/-0.15$ dex uncertaintes in the classification.

Of the $100 \mathrm{SF}$ galaxies within the redshift range useful for the red diagnostics, 81 have spectra with all four lines detected, while for the blue diagnostics the ratio is 63/91. As expected from Fig. 2, a non-negligible number (14) of optical AGN (i.e., those defined by line ratios) are in the SF galaxy sample.

For the red diagnostics, 18 objects are found in the "composite galaxy region": by applying the same procedure described in Sect. 3.1 to estimate the relative contributions of AGN and star formation to the radio flux, for these objects we obtained on average of $\sim 2.5$. For the non-passive and passive AGN samples, the diagnostics are inconclusive, because of the lack of detected lines for estimating the ratios. The blue and red diagnostics infer a different ratios of the number of detected AGN to star-forming galaxies. This is related to the different efficiencies of the two diagnostics in detecting AGN, as noted in Bongiorno et al. (2010) (see their Figs. 3 and 4). In particular, the blue diagnostics classify a large number of X-ray emitting AGN as star-formimg galaxies, reinforcing therefore the need of new AGN-star forming division methods.

Another method for differentiating between AGN and SF galaxies is the use of the $D_{n}(4000)-L_{1.4 \mathrm{GHz}} / M_{\text {star }}$ plane (Best et al. 2005), where the age of the galaxy stellar population (estimated by $D_{n}(4000)$ ) is compared with the specific star formation rate derived from the radio luminosity. The division line adopted by Best et al. (2005) is 0.225 above the $D_{n}(4000)$ value of the track defined by a 3 Gyr exponentially decaying star formation. In Fig. 5, we show our samples in this plane. The number of AGN below the division line is 5 (out of 104), while $~ 50 \%$ of our SF galaxies are above the Best et al. (2005) dividing line and would be classified as AGN on the basis of this criterion. These galaxies are probably in a transition region, where our AGNinduced emission objects are mixed with the SF ones.

The Smolčic et al. (2008) PCA-based classification is another, complementary way of classifying AGN and star-forming objects. Of the 129 AGN defined in Smolčic et al. (2008) that are in common with the zCOSMOS sample, 66 are star-forming excess galaxies in our classification scheme (and therefore AGN), while 9 out of 97 of the Smolčic et al. (2008) star-forming radio galaxies are in our AGN locus. The fraction of AGN that have $\log (S S F R)<-10.5$ is $\sim 60 \%$. A similar percentage of star forming excess was found in the AGN sample defined by Bardelli et al. (2009), where a spectrophotometric division was adopted. The apparently large difference between our own and the Smolčic et al. (2008) method is largely due to the fact that optical AGN and composite galaxies are included in the AGN sample in Smolčic et al. (2008) and not in our scheme.

In fact, by applying our diagnostics to the Smolčic et al. (2008) sample, we confirm that the contamination of their AGN and star-forming sample is $\sim 17 \%$ and $\sim 10 \%$, respectively, consistent with what is estimated in their paper.

A general conclusion of this comparison is that our approach, which relies entirely on the comparison of observed radio power with the expected power due to the star formation, is more conservative (and therefore not complete) in selecting radio AGN

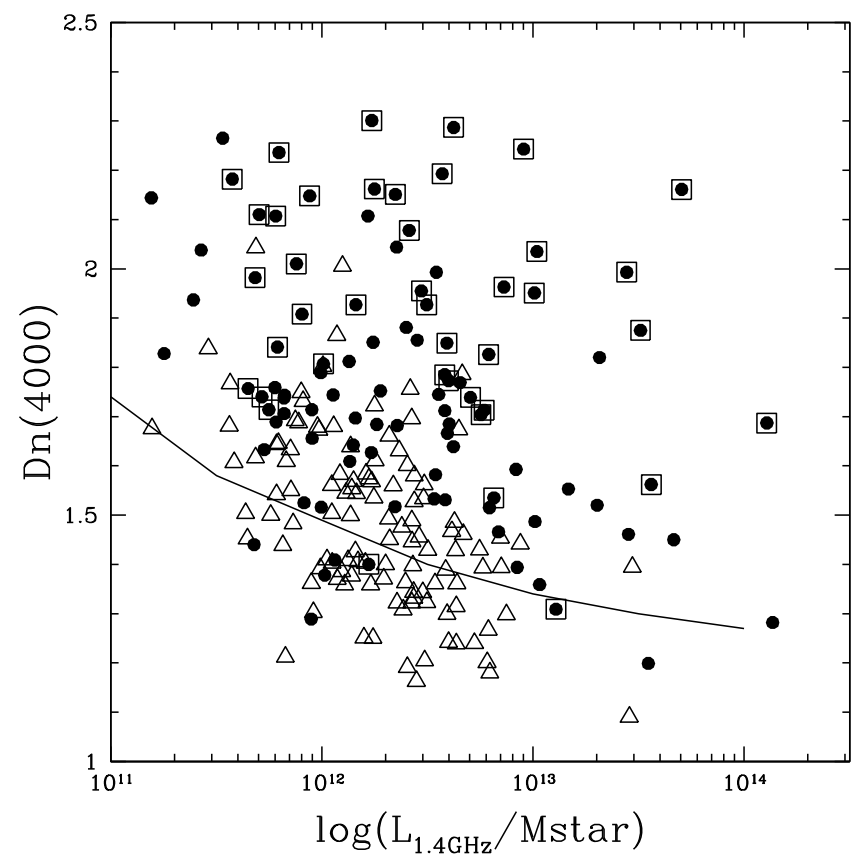

Fig. 5. $D_{n}(4000)$ versus $L_{1.4 \mathrm{GHz}} / M_{\text {star }}$ plane: triangles are our starforming galaxies and circles our AGN sample. Open squares indicate the passive AGN.

than other methods. We remind that our method was developed to classify as AGN those objects for which the radio flux is dominated by the central engine.

\section{Differences between radio-loud and control samples}

\subsection{Spectral differences between radio-loud and control samples}

The most basic approach is to construct composite spectra of the samples.

In Figs. 6 and 7, we show the composite spectra of the passive and non-passive radio AGN and star-forming galaxies and their control samples. We plot the difference between the radio and control sample composite spectra.

For passive AGN, there is no significant difference between the composite spectra of the radio loud and control sample, implying that there is no obvious AGN signature in their optical spectra. There is only an indication that the radio spectra are redder than the control sample.

The spectra of both the non-passive AGN and control sample objects exhibit significant [OII] emission lines, as well as absorption lines (like $\mathrm{Ca} \mathrm{H}+\mathrm{K}$ and $\mathrm{Mg} 5175 \AA$ ) and relatively weak [OIII] and $\mathrm{H} \alpha$ lines.

In both spectra, $\mathrm{H} \beta$ is only in absorption. All of these spectral features imply that these galaxies are of relative early type. All galaxies from the non-passive AGN and control sample are indeed classified by Zucca et al. (2009) as spectrophotometric type 1 and 2 (roughly corresponding to Sa-Sb). However, non-passive AGN tend to be of even earlier spectrophotometric types than the control sample. Galaxies in the radio sample have stronger [OII] equivalent widths ( $15 \AA$ versus $7 \AA$ ) with respect to the control sample.

For the star-forming galaxies, the difference between the two composite spectra is negative in [OII] and positive in $\mathrm{H} \alpha$, implying that in radio-emitting star-forming galaxies a larger amount 

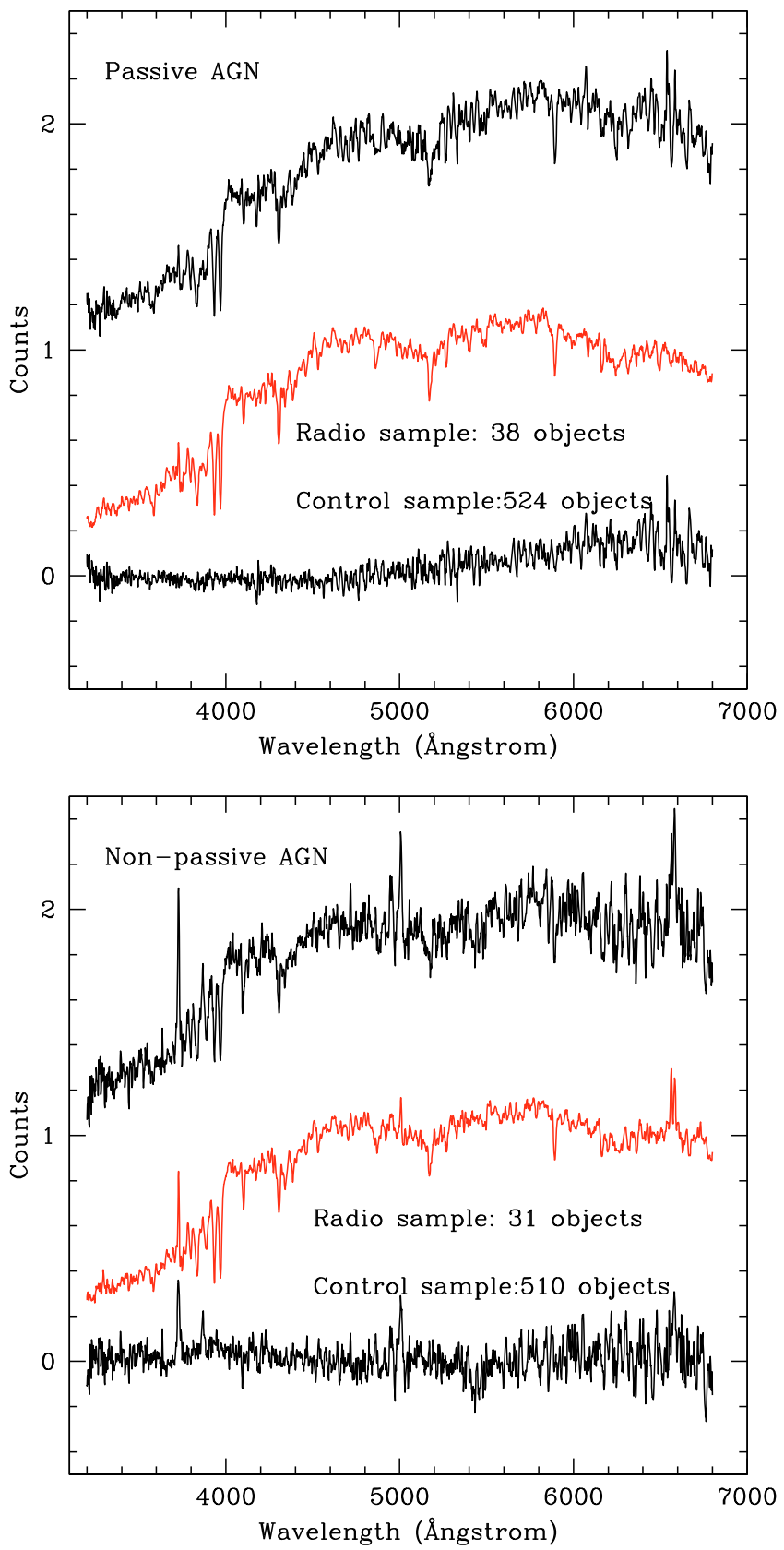

Fig. 6. Composite spectra for the radio-emitting AGN (in black, upper spectrum) and the control sample (in red, middle spectrum). The upper panel refers to passive AGN and the lower panel to non-passive AGN. The spectrum of radio sources is shifted for clarity by 0.9 in the $y$-axis. At the bottom of the figures, we plot the difference between radio-emitting objects and control sample (lower spectrum).

of line extinction (from dust) is present. This is confirmed by the continuum of radio-emitting galaxies being redder than the control sample. This is related to the differences in colors already found by Bardelli et al. (2009) for VVDS radio galaxies and present also in our VLA-zCOSMOS sample.

Additional information about the spectral features can be derived from the equivalent width distribution of the lines. We checked this point by examining the distribution of the equivalent width of the [OII] $3727 \AA$ line and the $4000 \AA$ break (as measured in Mignoli et al. 2009). There are no significant differences between the distributions of these quantities for passive AGN and their control sample.

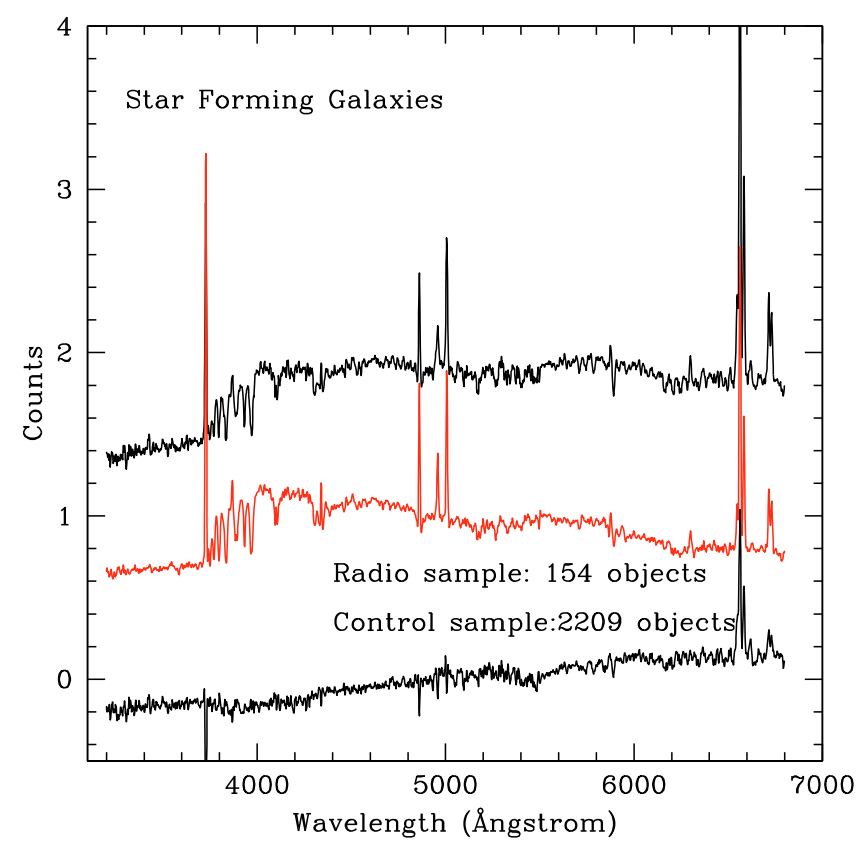

Fig. 7. Same as Fig. 6 but for the radio-emitting star-forming sample and its control sample.

Viceversa, the radio-detected star-forming objects have

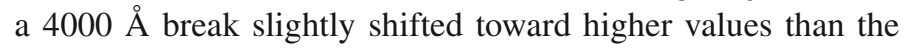
corresponding control sample. Given that the stellar mass distribution of the radio-detected star-forming galaxies and control sample is different and that our data exhibit a stellar mass$4000 \AA$ break relation, this difference probably is likely caused by the different stellar masses sampled (see Sect. 4.2). The differences disappear when considering objects of stellar mass $>2 \times 10^{10} M_{\odot}$, for which the mass distributions of the radio star-forming galaxies and their control sample are identical (see Sect. 4.2).

The differences are more subtle for the non-passive AGN sample (Fig. 8). The radio detected objects have higher values of the $4000 \AA$ break than the control sample and lower values of the equivalent width of the [OII] $3727 \AA$ line (the probability inferred by the KS test that the two distributions originate from the same distribution is $\sim 0.005$ ).

The last question is whether the galaxies in the control sample of non-passive AGN show any sign of AGN activity in their emission lines. In Fig. 9, we show the diagnostic diagrams for this sample. The blue and magenta circles correspond to values obtained from the composite spectra of non-passive AGN and their control sample, respectively. Considering galaxies with either all lines detected or only one line detected of the considered ratio, $\sim 50 \%$ of this sample consists of optical AGN or upper limits consistent with that of AGN. We note that this fraction is higher in the red, low-redshift diagram. These fractions are broadly consistent with those of the radio sample, although the number of objects with all detected lines or meaningful upper limits is only 20 for the radio detected non-passive AGN sample.

We note that the points corresponding to the composite spectra are not in the middle of the distribution of the ratios of single galaxies. This is because the composite spectra are also obtained by considering all objects not plotted in Fig. 9 (which are $\sim 48 \%$ and $27 \%$ of the samples in the blue and red diagram, respectively) because of the lack of detected lines. Therefore, 
S. Bardelli et al.: Properties and environment of radio-emitting galaxies
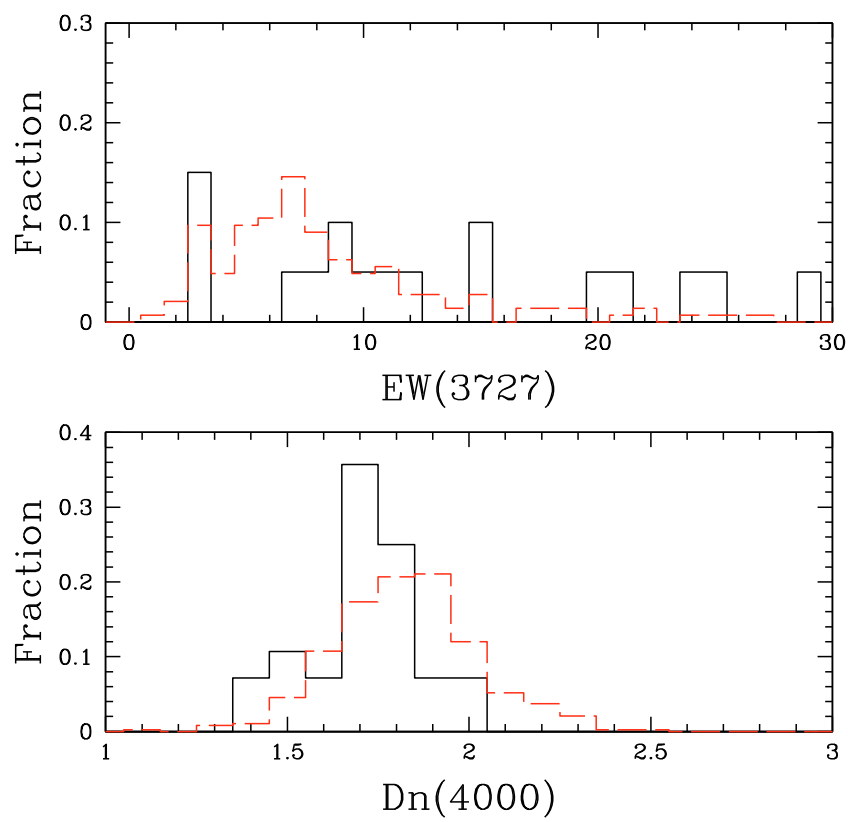

Fig. 8. Equivalent width and $D_{n}(4000)$ distributions of the radio and control sample for the non-passive AGN. The dashed, red line is for the control sample and the black solid line is for the radio sample.

composite spectra are not expected to have the average ratios of the plotted points.

\subsection{Stellar mass distributions}

Before studying the effect of the different environments on the radio galaxies, it is necessary to verify the consistency between the stellar masses of the various samples of radio galaxies defined in the previous sections with those of their respective control samples. Given the mass-density relation (Scodeggio et al. 2009; Bolzonella et al. 2010), a difference in the stellar mass distribution may induce a difference in the observed overdensity distributions that would not be related to a AGN or starformation mechanism.

To consider a homogeneous population at all redshifts, we selected galaxies and radio sources within an optical luminositycomplete, volume-limited sample at $z<0.9$. To take into account the observed optical luminosity evolution (Zucca et al. 2009), the absolute magnitude limit was defined to be $M_{B}=-19.8-z$. This limit in absolute magnitude corresponds to the zCOSMOS limit in apparent magnitude $(I<22.5)$ at $z=0.9$. In Fig. 10 , we show the observed mass distibution of the passive AGN and their control sample.

For the control sample, since we have a volume-limited sample, we plot the stellar mass histogram. Since the mass completeness limit changes with redshift (from $\sim 10^{9} M_{\odot}$ at $z \sim 0.3$ to $\sim 5 \times 10^{10} M_{\odot}$ at $z \sim 0.9$ ) (see Pozzetti et al. 2010), our observed stellar mass distribution shows an increasing incompleteness at decreasing stellar mass. Assuming that the incompleteness is the same for the control sample and the radio sample, only the relative comparison between the control and radio sample is relevant here. The radio sample, which also has a limit in radio flux in addition to the limit in apparent magnitude, needs to be corrected for $V_{\max }$.

The first result is that all radio-emitting objects have $\log \left(M_{\text {star }}\right)>10.7$, while the control sample has a broader mass
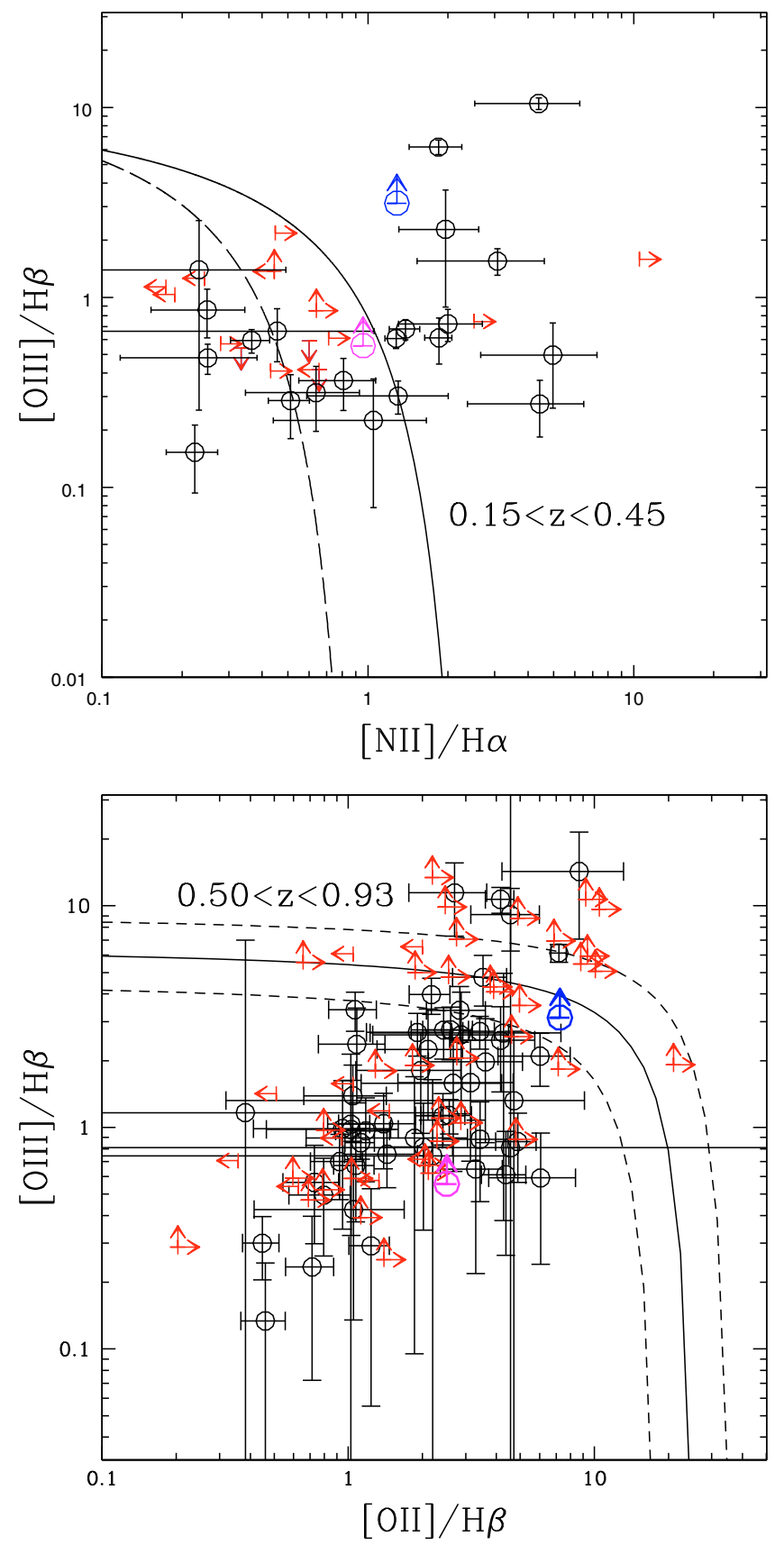

Fig. 9. Line diagnostics for the control sample of the non-passive AGN sample. The blue and magenta points correspond to the values obtained from the composite spectra of non-passive AGN and control sample respectively.

distribution. For this reason, we limit the control sample to $\log \left(M_{\text {star }}\right)>10.7$ in the following analysis.

We note that the ratio of the stellar mass distribution of radioemitting passive AGN to its control sample is rather constant with stellar mass at a value of $\sim 20 \%$. This is apparently inconsistent with the results of Smolčic et al. (2009a), for which the ratio of the stellar masses of radio AGN to that of its control sample rapidly increases with stellar mass. The difference could be understood by considering that we study only a specific class of AGN and apply a significantly high cut in absolute magnitude to create a volume-limited sample at the depth of zCOSMOS. On the other hand, Smolčic et al. (2009a) considered volumelimited samples in terms of the radio limit (i.e., fixing a limit in 


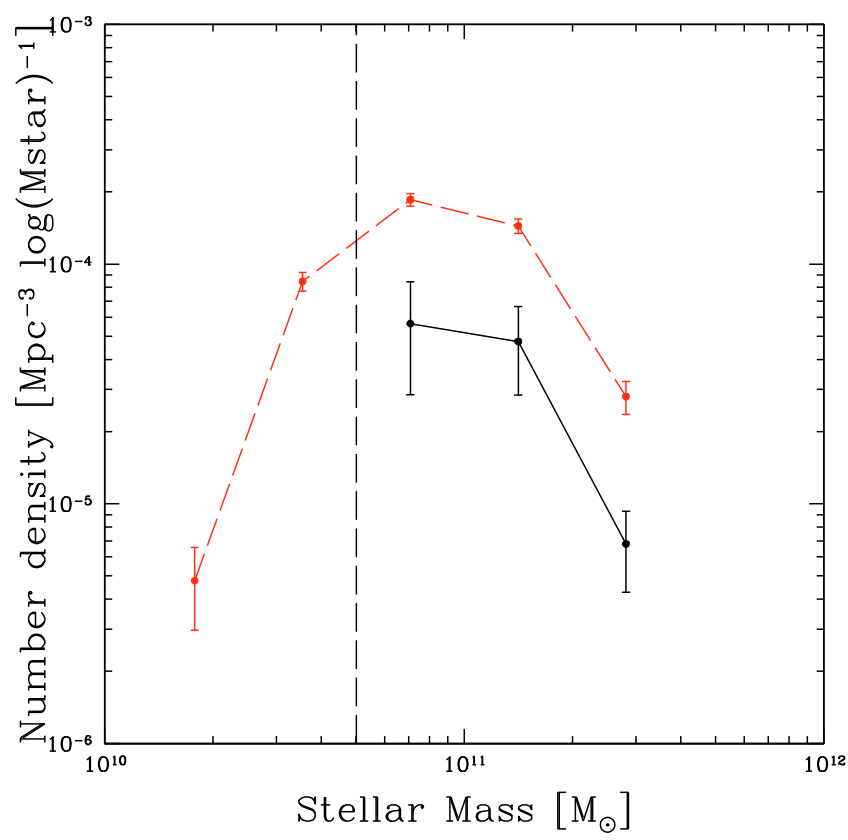

Fig. 10. Stellar mass distribution of the passive AGN (black, continous line) compared with its control sample (red, dashed line). The vertical dashed line indicates the adopted stellar mass cut.

the radio luminosity) and corrected for $V_{\max }$ the optical incompleteness. Their apparent magnitude (and consequently absolute magnitude range) limit is deeper than that of the zCOSMOS survey. Considering all AGN (passive+blue) and avoiding the absolute magnitude limit, we derive similar values of the these ratios as Smolčic et al. (2009a).

For non-passive AGN, the ratio of the two stellar mass distributions (radio-detected and control sample) is $\sim 5 \%$ for masses $<10^{11} M_{\odot}$ (see upper panel of Fig. 11), while at higher masses the ratio is $>25 \%$. In this case, we note that we are able to infer the mass dependence of the AGN activity.

For star-forming galaxies, the lower panel of Fig. 11 indicates that the the two stellar masses distributions are the same for masses higher than $2 \times 10^{10} M_{\odot}$ and differ for lower masses, consistent with a similar plot in Bardelli et al. (2009).

We interpret this trend as implying that the stellar mass distribution of radio-detected star-forming galaxies is the convolution of the stellar mass function of these objects with the star-formation rate distribution at fixed stellar mass or absolute magnitude (see Bardelli et al. 2009). The mean star-formation rate increases with mass, but the distribution has significant tails. At lower stellar masses, an increasing number of objects with low star formation rate is below the flux limit of the radio survey. More specifically, the lower limit to the star formation rate is set to $\log \left(S F R / M_{\text {star }}\right) \sim-10.5$ from Fig. 3, which corresponds to $\log \left(M_{\text {star }}\right)=10.3$ (the "completeness limit") to $\sim 0.6 M_{\odot} \mathrm{yr}^{-1}$, similar to the minimum observed star-formation rate. For lower masses, the limit is lower than the minimum observed starformation rate.

For the density analysis, we limit the control sample to $\log \left(M_{\text {star }}\right)=9.7$ for the star-forming galaxies and to $\log \left(M_{\text {star }}\right)=$ 10.4 for non-passive AGN. These limits correspond to the minimum mass detected in the radio samples.
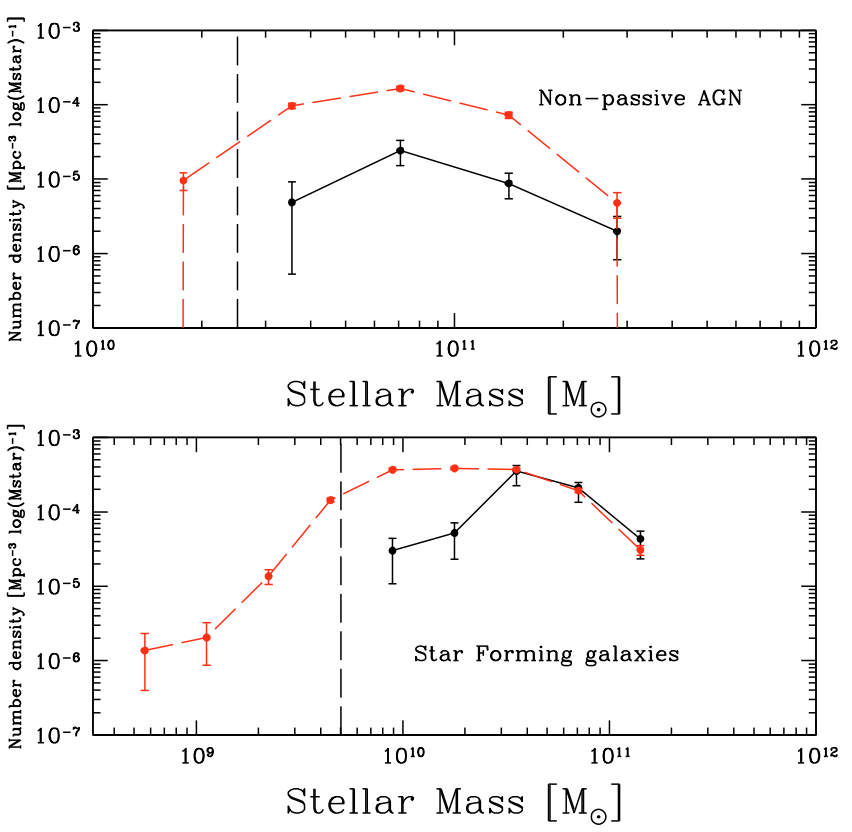

Fig. 11. Upper panel: stellar mass distribution of the non-passive AGN (black, continous line) compared with its control sample (red, dashed line). Lower panel: same but for star-forming galaxies.

\section{Environmental effects}

\subsection{Method}

To define our radio and control sample consistently, we adopted the limits reported in Table 1 . We computed the density distributions by considering all objects at $z<0.9$ and brighter than an "evolving" absolute magnitude limit of $M_{B}<-19.8-z$, where $z$ is the galaxy redshift. This evolving limit was introduced to take into account roughly the luminosity evolution of the galaxies as determined from the luminosity function estimated by Zucca et al. (2009, 2006), and therefore to consider approximately the same population at all redshifts. In the case of the passive AGN sample, we also applied a cut in stellar mass at $\log \left(M_{\text {star }}\right)>10.7$, for the non-passive galaxies at $\log \left(M_{\text {star }}\right)>10.4$ and for the star-forming galaxies at $\log \left(M_{\text {star }}\right)>9.7$ (see Sect. 4.2).

\subsection{Redshift distribution}

The most basic approach to assessing a possible dependence of the properties of radio galaxies on environment is to compare their redshift histogram with that of the control sample. In Fig. 12 (upper and lower panels), the normalized histograms of the radio sources (solid, black line) and the control sample (dashed, red line) are shown.

In this figure, we apply a lower limit to the radio luminosity to the radio sample at $\log L_{\text {Radio }}\left(\mathrm{W} \mathrm{Hz}^{-1}\right)>23$ to obtain a volume-limited sample also in terms of radio luminosity. Variations in the ratio of the number of radio galaxies to those in the control sample found in correspondence to peaks or valleys of the galaxy distribution would indicate a different environment distributions of the two populations. Smoother variations as a function of redshift would imply an evolution of sources.

In the upper panel we consider all AGN (non-passive + passive), i.e., those radio sources with star-formation rate excess and, for the control sample, all galaxies with $\log (S S F R)$ (optical) $<-10.5$. In the middle panel, we show the 

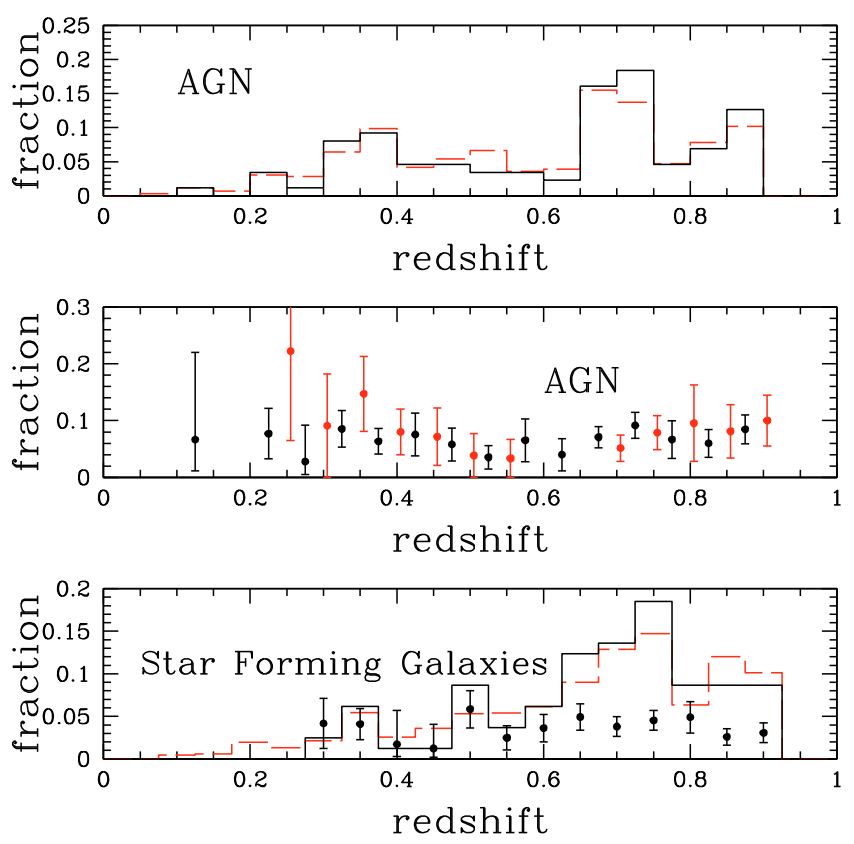

Fig. 12. Upper and lower panels: fractional redshift distributions of our samples. Red dashed histogram: control sample. Black solid histogram: radio sample. Histograms have been normalized to the number of objects. Upper panel: AGN sample (passive+non-passive). In the middle panel, we show the ratio of the number of radio AGN to that of the control sample. Red points represent the fraction of radioloud passive AGN (shifted for clarity by 0.03 in the $x$ axis). Lower panel: star-forming galaxy redshift histograms and their relative ratios (black points).

ratio of the two histograms to visualize better any redshift dependence. Errors correspond to the $1 \sigma$ confidence level. Black points correspond to the entire AGN sample, while red points refer to passive AGN. For clarity, the red points have been shifted by +0.03 along the $x$ axis. In the lower panel we show the redshift distribution of star-forming galaxies.

The number ratios of both AGN and SF radio samples to their respective control samples are rather constant with redshift. This implies that the radio samples follow the same largescale distribution as the control samples and therefore no large segregation with density is present on the largest scales. For the two classes of AGN taken separately, the ratio values are around 0.07 for the non-passive AGN sample and 0.06 for the passive AGN sample.

The redshift histograms explore densities on the largest scale possible for our redshift survey, ranging from $\sim 12 \mathrm{Mpc}$ at $z \sim 0.3$ to $\sim 21 \mathrm{Mpc}$ at $\sim 0.5$. In the following, we explore smaller scales using the 5 th nearest neighbor density estimator.

\subsection{The nearest neighbour determined overdensity distribution}

We used the densities estimated from the distance to the 5 th nearest neighbor (Kovač et al. 2010) and the volume-limited sample as a tracer of the density field. In this case, the tracers are galaxies of absolute $B$ band magnitude brighter than $-19.5-z$ to redshift 0.7 , while for redshifts between 0.7 and 1 , the tracers are galaxies with $M_{B}<-20.5-z$. This corresponds to overdensities computed on average scales of $\sim 2.4 \mathrm{Mpc}$, thus not much larger than the typical extent of a cluster. We also checked the results obtained with the other tracers described in Kovač et al. (2010), but the results did not change. Among the various available weighting schemes, we used the densities weighted by the stellar masses of the galaxies, but the results did not change either when using the no-weight (i.e., number weighted) estimator.

In Fig. 13, we compare the overdensity distribution of the three radio samples (black solid histograms) with those of their respective control samples. In the upper left panel, we present the total AGN sample. In this case, we recall that the radio sample comprises all radio sources with a star-formation excess and the control sample comprises all objects with $\log (S S F R)$ (optical) $<$ -10.5 . The two distributions differ at about the $2 \sigma$ level on the basis of the K-S test.

A much stronger statistical signal of difference (as evaluated by the KS test probability) is obtained by considering only the passive AGN (upper right panel). Here, we note a lack of radio sources at low overdensities $(<3)$ and an excess at high overdensities $(>10)$. The signal for the whole AGN sample is produced entirely by passive AGN. If we select only the non-passive AGN sample, the KS test does not find any significant difference (lower left panel). In Fig. 14, we plot the same data but now as the ratio of the number of radio-emitting passive AGN to the control sample. As can be seen, the ratio steadily increases with density from 0.02 to 0.2 .

There is the possibility that this behavior is caused by the observed ratio of the stellar mass functions of radio to the control sample that vary between 0.02 (in the bin at lower mass in Fig. 10) to $\sim 0.2$ (in the highest bin). We note that in Fig. 10 we presented the $V_{\max }$-corrected mass distributions. In other words, spurious density differences caused by the stellar mass-density relation could be induced both by true differences between the stellar mass fucntions of the radio and the control samples, and also by the fact that the observed mass distributions are different (despite the mass functions themselves being similar) merely because of the flux limit.

To check this point, we recomputed the control sample overdensity histogram of the upper right panel of Fig. 13 by weighting all the masses by the ratio of the observed mass distribution of the radio to that of the control sample. The results changed by a negligible amount. We searched for a possible dependence on the redshift of the difference between the environments of radio and control sample. The differences between radio and control samples remain significant, although the density distributions of both samples have higher density tails at lower redshift, which is consistent with the evolution of galaxies and of the overdensities. We conclude that no redshift evolution is present in the density-radio-emission relation.

Another apparently significant difference in the environmental distribution of radio and control galaxies is found for the starforming galaxies. The radio-emitting star-forming galaxies reside in systematically higher densities than the control sample. However, we note also that for star-forming galaxies the stellar mass functions for the radio and control sample differ (lower panel of Fig. 11).

We repeated the previous procedure to correct the control sample for the stellar mass distributions ratios and we found that the significance of the difference disappears. We computed the $\chi^{2}$ probability of the two distribuions. We can not use the KS test because of the binned mass ratios assumed for the correction: the probability that the two distributions are the same increases from $\sim 0.05$ to $\sim 0.90$.

Therefore, we conclude that most of the difference between the overdensity distributions of the radio and control starforming galaxy samples is caused by the different stellar mass distributions sampled by the radio and control samples (higher 

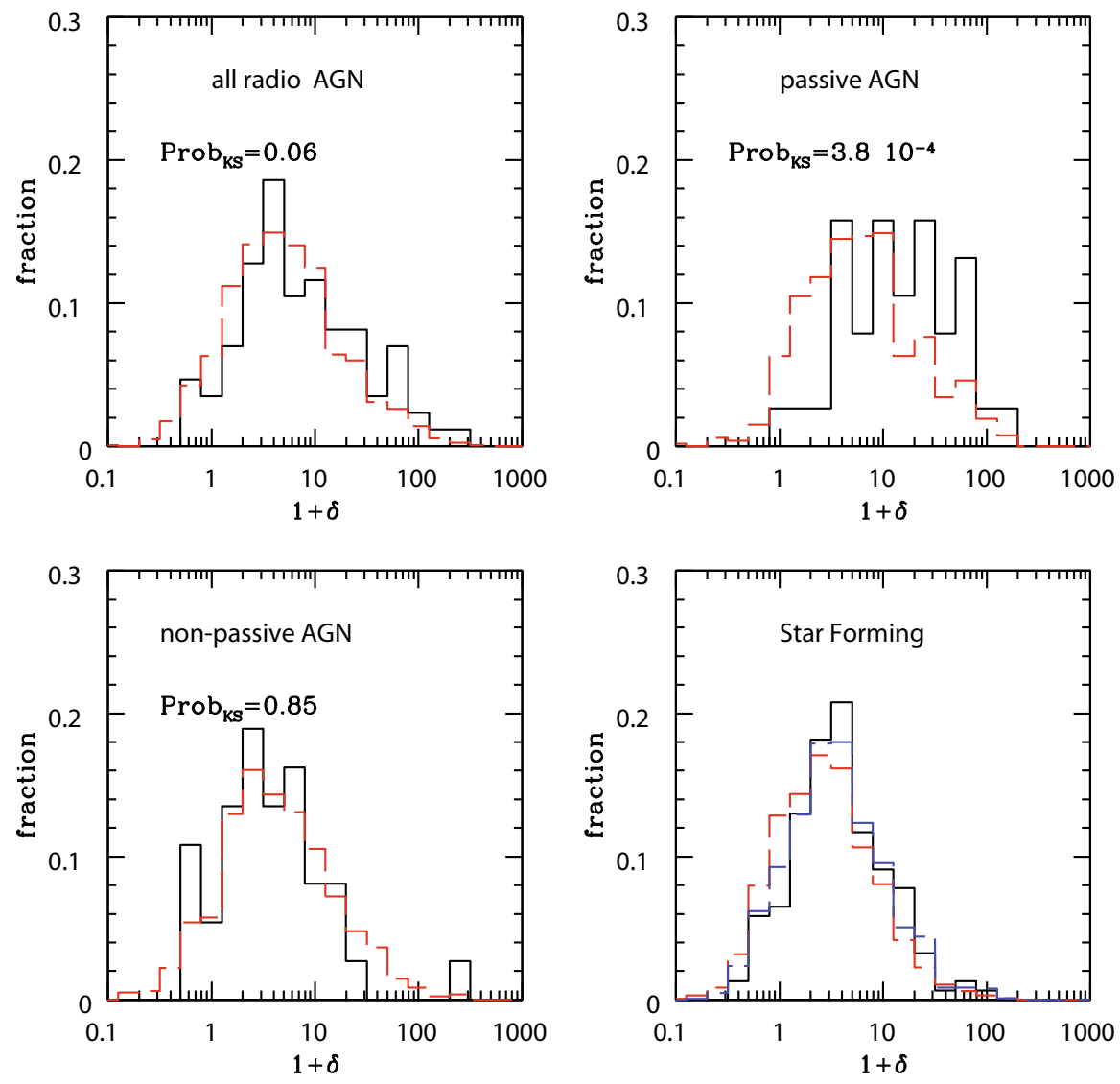

Fig. 13. Black histograms: overdensity distribution of the radio sources, Red dashed histograms: control samples. Upper left panel: total AGN sample. Upper right panel: passive AGN sample. Lower left panel: non-passive AGN. Lower right panel: star-forming galaxies. The blue histogram corresponds to the corrected control sample.

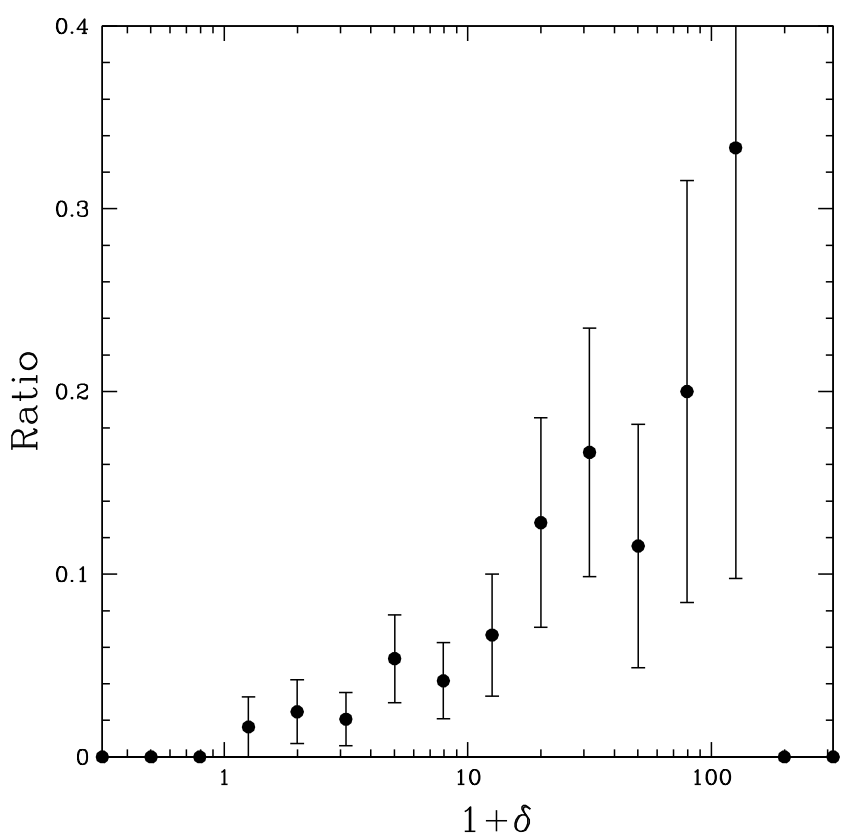

Fig. 14. Ratio between the radio and control sample of passive AGN as a function of the density.

masses reside in higher densities) and not by the physical role of the environment on the radio emission.

All results showed in this section are stable also using the 10th and 20th nearest neighbor, which correspond to scales of 3.5 and $5 \mathrm{Mpc}$, respectively.

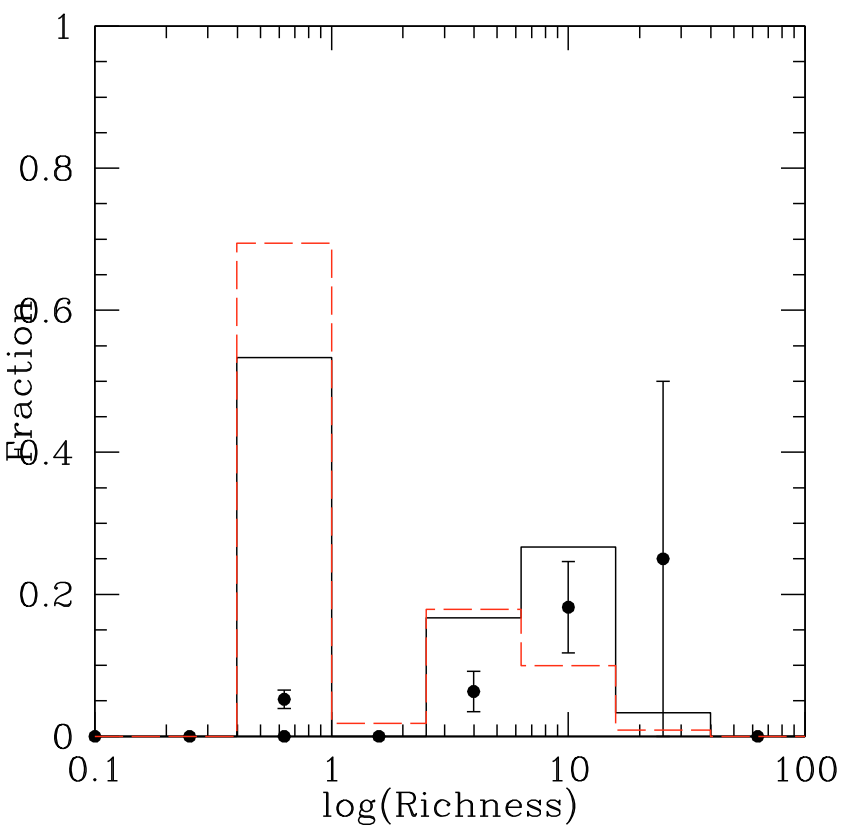

Fig. 15. Fractional distribution of richness of the groups where radio passive AGN (solid line) and galaxies of the control sample (red dashed histogram) reside. Circles with error bars represent the ratio between the numbers of objects of these two samples.

\subsection{Groups of galaxies}

In Fig. 15, we show the ratio of the number of radio passive AGN to the control sample as a function of group richness. The 
fraction of passive galaxies that are AGN in the isolated galaxies sample is $0.052(16 / 307)$, while the fraction in groups is $0.104(14 / 135)$, i.e., the difference is not statistically significant. However, we note that $2 / 3$ of the control sample galaxies are isolated and $1 / 3$ reside in a group, while the radio passive AGN are nearly equally distributed between the two classes.

However, if we limit the group sample to those of richness $>4$ (for which the contamination by false groups is small), the fraction of radio passive AGN becomes 0.138, and the significance of the difference between the fraction of passive AGN among isolated galaxies and that in groups becomes $2.15 \sigma$. The overall distributions of radio and control samples has a significance derived from the K-S test of $8 \%$ of being drawn from the same distribution. Therefore, there is a small statistical signal due to an increase of the fraction of radio objects with richness.

\section{Properties of the passive AGN}

In the previous section we showed evidence that passive AGN have an increasing probability of being radio sources in denser environments. Here, we explore whether other properties of single passive AGN also change as a function of local density. In practice, we divided the sample of passive AGN into two bins joining at a value of $1+\delta=10$, and compared a number of quantities for the two subsamples. First of all, we computed the two separate luminosity functions, We note that it is only possible to consider differences in shapes, since the relative normalizations are influenced by the volume occupied by the various overdensities within the survey volume, which is difficult to control. For this reason, we compared only the shapes that were indistinguishable. This is not unexpected because already Ledlow \& Owen (1996) did find that the radio and field luminosity functions have similar shapes.

In Fig. 16 (upper panel), the radio power-stellar to mass ratio is plotted. The dashed, red lines correspond to the high density sample and the solid, blue line to the low density one. Each ratio has been weigthed by its $V_{\max }$ following Bardelli et al. (2009). At a given stellar mass, it appears that the radio luminosity of the AGN is significantly higher in dense environments.

In the lower panel of Fig. 16, we plot the radio luminosity versus stellar mass. We note that the difference between the two panels is that in the upper one the radio-luminosity to stellarmass ratio is corrected by $V_{\max }$, while in the lower panel we plot the observed points. It appears to be a transition luminosity $\left(\right.$ at $\left.\log L_{\text {radio }}\left(\mathrm{W} \mathrm{Hz}^{-1}\right)=23.5\right)$, below which the objects are about equally distributed between low and high density regions, while above this luminosity $\sim 80 \%$ of radio sources reside in high density regions. There is also an indication that below the luminosity threshold, a correlation between mass and luminosity is present (confirmed by a Spearman rank correlation test), which disappears at higher luminosities.

In Fig. 17, we show the black-hole mass distribution of the passive AGN (black) and the control sample (red). We estimated the black-hole mass using the prescription of Marconi \& Hunt (2003)

$\log _{10}\left(\frac{M_{\mathrm{BH}}}{M_{\odot}}\right)=8.21+1.13\left[\log _{10}\left(\frac{L_{K}}{L_{\odot}}\right)-10.9\right]$

where $L_{K}$ is the $K$-band luminosity of the galaxy. We show the results for the local $M_{\mathrm{BH}}-L_{K}$ relation, because Peng et al. (2006) claim that this relation does not change with redshift. We also corrected the Marconi \& Hunt (2003) formula in a similar way to Smolčic et al. (2009a) for the evolution in luminosity of a
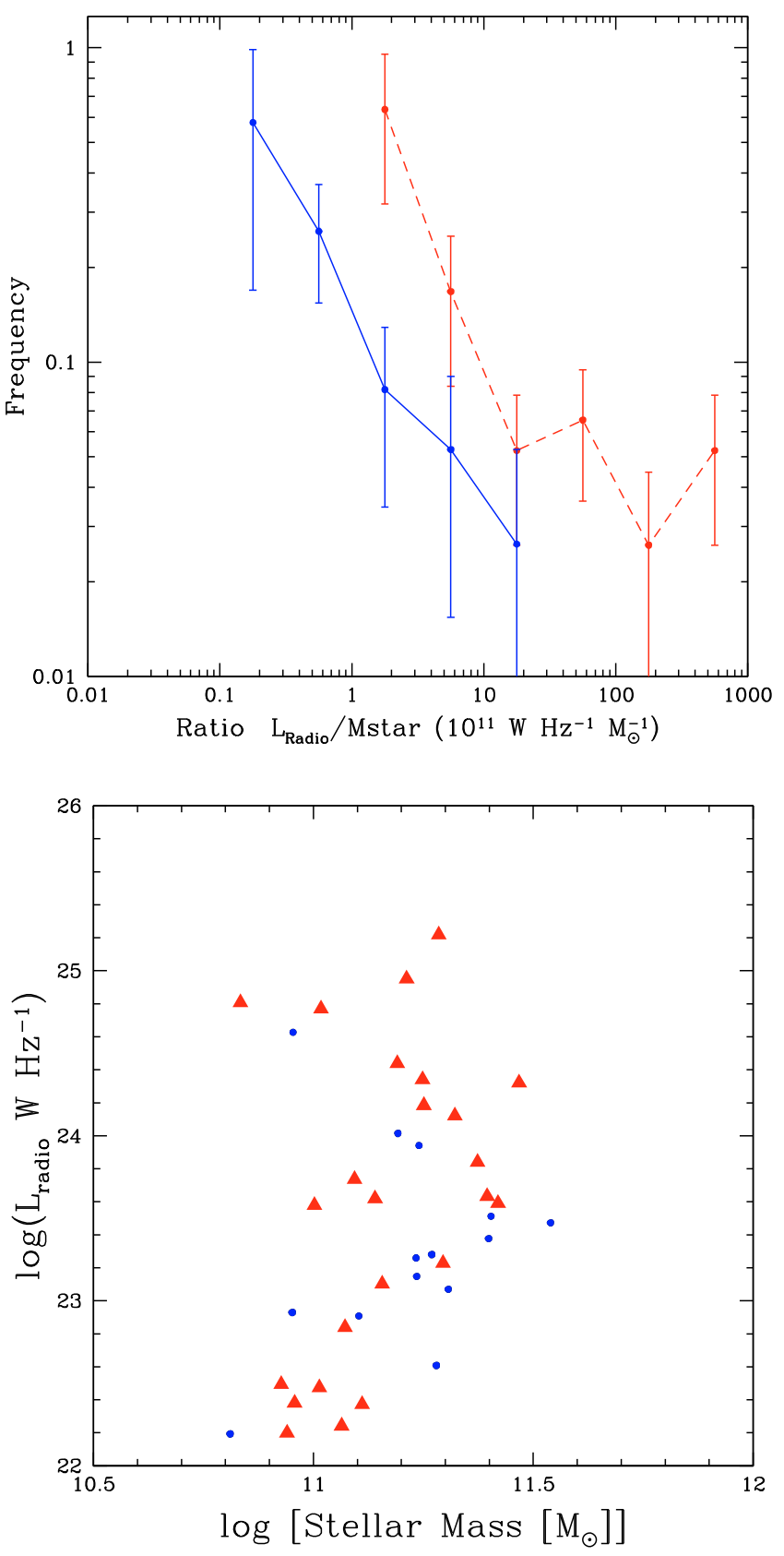

Fig. 16. Upper panel: distribution of $L_{\mathrm{Radio}} / M_{\text {star }}$ for passive radio AGN in two bins of overdensity (red dashed line for high and blue continous line for low overdensity). Lower panel: radio luminosity versus Stellar Mass for the same samples. Red triangles represent high densities and blue points the low densities.

passive population (see Hopkins et al. 2006) but our results did not change.

There is no significant difference between the mass distribution of high and low density AGN. The only detectable difference is between the distributions of high and low density control sample (with a KS probability for the two distribution is of $\sim 0.005$ ). If we plot the radio luminosity versus the black-hole mass (not presented here), we obtain a plot similar to the lower panel of Fig. 16 but with the stellar mass replaced by the blackhole mass. This is reasonable considering the relation between stellar mass and $K$-band luminosity.

Our general conclusion is that the main difference between passive AGN in low and high density environments is in terms 


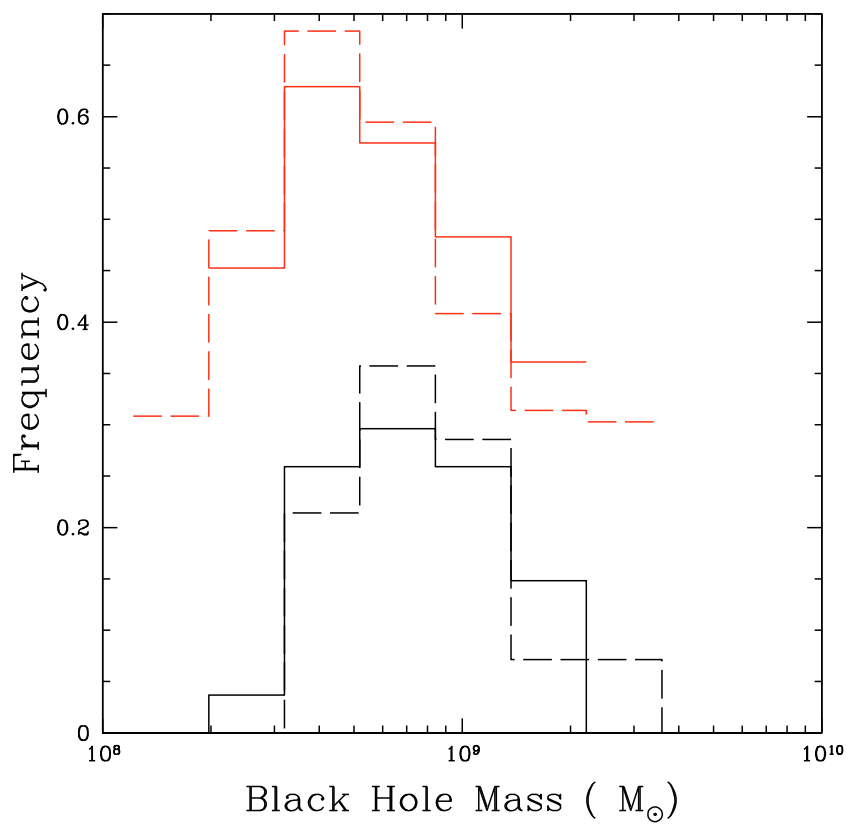

Fig. 17. Black solid histogram: high density $(1+\delta>10)$ black-hole mass distribution for passive AGN. Black, dashed histogram: same distribution but for objects with $(1+\delta<10)$. In red, the distribution of masses for the control sample, shifted for clarity along the vertical axis by 0.3 .

of the radio power, which is, given the similarity in the black hole masses, related either to the host galaxies or environment structure.

\section{Discussion and conclusion}

The study of the environment of radio galaxies requires first a division of the radio sources on the basis of their emission mechanism, because AGN and star-forming galaxies may be influenced differently by their local density. To do this, we compared the star-formation rate estimated from the spectral energy distribution with that derived from the radio luminosity. We found a clear correlation between these two quantities for star-forming galaxies. Therefore, we defined as AGN those objects for which the radio star-formation rate is significantly higher than the optical one. In other words, these objects have a radio luminosity significantly higher than that predicted by their star formation. We apply a stringent lower limit cut at which the AGN radio emission is more than an order of magnitude higher than the star-formation emission in the same galaxy. In this way, we excluded from our radio-based AGN sample those AGN defined on the basis of their optical emission lines, such as Seyfert 2 and LINERs, for which the ratio of AGN to star-forming radio emission is lower than the adopted threshold.

Since AGN are hosted preferentially by optical early-type galaxies and star-forming objects are basically spiral galaxies, we expect to recover at least the optical morphology-density relation (Dressler 1980), which is not related to the radio emission. Therefore, to investigate whether there is an environmental effect on the radio emission, we defined control samples tat could be compared with the AGN and star-forming radio samples. To achieve this, we studied the positions of radio sources and zCOSMOS galaxies in an infrared color-specific star-formation rate plane. We defined three populations: passive AGN, nonpassive AGN, and star-forming galaxies. The division between passive and non-passive is reminescent of the low and highexcitation dichotomy described in Smolčic (2009) and probably corresponds to two different types of accretion processes (hot and cold, respectively). The two processes are expected to depend differently on the environment (Hardcastle et al. 2007). The radio luminosity functions of the passive and non-passive AGN are similar in shape but not in luminosity range. Nonpassive AGN stop at $\log L_{\text {radio }}\left(\mathrm{W} \mathrm{Hz}^{-1}\right)=23.4$, while the passive $A G N$ reach $\log L_{\text {radio }}\left(\mathrm{W} \mathrm{Hz}^{-1}\right)=25.2$.

Finally, almost all our radio objects belongs to the FR-I class (defined by Ledlow \& Owen 1996), and the sample does not include the broad line AGN class. We therefore focussed mainly on objects triggered by gas accretion and did not consider other mechanism such as e.g., mergers.

Passive AGN: this population of AGN is hosted by red galaxies. Using the morphological classification derived from HST/ACS images by means the ZEST algorithm (Scarlata et al. 2007; Tasca et al. 2009), we found that $\sim 85 \%$ of both passive AGN and their relative control sample are classified as early type (i.e., bulge dominated). The spectra of these galaxies with and without radio emission are indistinguishable and the ratio of their stellar mass functions is rather constant at $\sim 0.2$ for masses higher than $5 \times 10^{10} M_{\odot}$. This value can be interpreted as corresponding to the average fraction of time during which the central engine is switched on. We note that our ratio was derived by comparing the mass functions: if we had used the observed number of galaxies, we would have found values more similar to those reported in Table 4 of Shabala et al. (2008) ( 0.5 at high masses, $\sim 0.15$ at intermediate masses, and $\sim 0.01$ at low masses).

This population exhibits a strong dependence on environment: the fraction of objects with radio emission increases from $\sim 0.02$ in underdense regions $(1+\delta<3)$ to $\sim 0.15$ in overdense regions $(1+\delta>10)$. Similarly, such trend is also found (but at a low significance level) as a function of richness of galaxy groups.

Studying the radio-luminosity versus stellar mass plot, we found that for luminosities lower than $\log P\left(\mathrm{~W} \mathrm{~Hz}^{-1}\right)=23.5$, there is a correlation between the two quantities, which disappears at higher luminosities. The correlation between stellar mass and radio luminosity is not unexpected because of the relation between the mass accretion onto a black hole and hot gas fraction in the "radio mode" (see Eq. (10) of Croton et al. 2006). As stated by Croton et al. (2006), the hot gas fraction is approximately constant for galaxies with $v_{\text {vir }}>150 \mathrm{~km} \mathrm{~s}^{-1}$ and therefore black hole luminosity scales with black hole mass, which correlates with the stellar mass. Interestingly, half of the objects in the low radio luminosity regime reside in low density environments, while the others are in overdense regions.

The correlation disappears for radio luminosities higher than $\log L_{\text {Radio }}\left(\mathrm{W} \mathrm{Hz}^{-1}\right)=23.5$. Almost all of these radio luminous objects reside in overdense regions. On the other hand, no difference is present between the black hole mass at high and low densities and it therefore seems that in richer environments the emission mechanism is more efficient than in low density regions.

Therefore, we can tentatively say that at low density the emission is determined by the host galaxy stellar mass and at high density by the host structure (group/cluster) in which the galaxy resides. The higher luminosities in denser environments could be caused by a greater fuel supply (due to the central cooling of the gas) and/or to outside the galaxy being a significantly high gas density to confine the radio jet. On the other hand, we already know that ellipticals at the center of galaxy groups are richer in hot gas than isolated and non-dominant ones (Helsdon et al. 2001) and this implies that a mechanism driving gas within dominant galaxies is or has been active. This can also justify 
the longer time spent by the central engine in the "switched on" mode, as suggested by the increasing ratio of the number of passive AGN to control sample as a function of density. It would be interesting to investigate whether the high density environment AGN have luminosities that correlate with the central cooling flow of the hosting groups, following the relation between radio luminosity and accretion mass found by Mittal et al. (2008). We note that our findings are consistent with those of both Best (2004), Mandelbaum et al. (2009).

Finally, the difference between the overdensity distributions of the radio-loud and control samples remains constant across the studied redshift range, implying that no significant evolution in this phenomenon is present.

Non-passive $A G N$ : this class is a less well-defined family. In principle, one can find in this class not only the AGN but also objects such as post-starburst galaxies. In this case, radio emission disappears on a time-scale of $10^{8}$ years, which could be longer than the disappearing time of the star-formation signatures in the optical. However, we expect that these objects are rare because of the implied short time-scales. In fact, only two of the radio detected post-starbursts of the Vergani et al. (2010) sample and one of their upper limits lie within our AGN region. This means that post-starburst galaxies contaminate our sample only at a level of $4 \%$. The non-passive AGN population occupies a small region of the infrared color-specific star-formation rate plot, which is approximately equivalent to the green valley defined in the optical bands. These galaxies are objects with spectrophotometric types earlier than $\mathrm{Sa}-\mathrm{Sb}$, and by applying standard diagnostics based on emission line ratios we found that $50 \%$ are classified as optical AGN and the remainder as star-forming galaxies. The ZEST morphologies of non-passive AGN are divided equally between early types and spirals (with $\sim 8 \%$ being irregulars), while the control sample has percentages of $\sim 60 \%$ and $40 \%$, respectively. The radio-emitting objects of this class follow the same environmental distribution of the corresponding control sample galaxies and do not exhibit any luminosity-density behavior.

Star Forming galaxies: the composite spectra of radioemitting star-forming galaxies is redder than the control sample and has a smaller [OII] and higher $\mathrm{H} \alpha$ equivalent width.

Considering that the radio-detected star-forming galaxies are objects where the star formation rate is on average higher than the undetected ones, this implies that the dust content is also higher. This increase in dust extinction with increasing star formation is already known (see e.g. Pannella et al. 2009) and could justify the relation between the emission lines and the radio SFR estimators shown in the lower panel of Fig. 1. The ZEST morphological classification shows that the radio-detected objects tend to be of earlier type than the control sample, as found for the spectrophotometric types.

The radio-emitting star-forming galaxies appears to have an apparent difference in density distribution with respect to the control sample, but we have shown that this is due to the different stellar-mass distributions of the two samples. Correcting the control sample for the fraction of radio-emitting objects, no differences in terms of environment are present between the radio and control sample galaxies. This is consistent with the conclusion of Kauffmann et al. (2004) that the star formation rate does not depend on the galaxy density for scales greater that that of the Megaparsec.

Acknowledgements. We acknowledge support from an INAF contract PRIN2007/1.06.10.08 and an ASI grant ASI/COFIS/WP3110 I/026/07/0.The VLA is operated by the National Radio Astronomy Observatory, which is a facility of the National Science Foundation, operated under cooperative agreement by Associated Universities, IIIC.

\section{References}

Baldwin, J. A., Phillips, M. M., \& Terlevich, R. 1981, PASP, 93, 5

Bardelli, S., Venturi, T., Zucca, E., et al. 2002, A\&A, 396, 65

Bardelli, S., Zucca, E., Bolzonella, M., et al. 2009, A\&A, 495, 431

Bell, E. F. 2003, ApJ, 596, 794

Best, P. N. 2004, MNRAS, 351, 70

Best, P. N., Kauffmann, G., Heckman, T. M., \& Ivezič, Z. 2005, MNRAS, 362, 9

Bekki, K. 1999, ApJ, 510, L15

Birzan, L., Rafferty, D. A., McNamara, B. R., Wise, M. W., \& Nulsen, P. E. J. 2004, ApJ, 607, 800

Bolzonella, M., Kovač, K., Pozzetti, L., et al. 2010, A\&A, submitted [arXiv:0907.0013]

Bondi, M., Ciliegi, P., Schinnerer, E., et al. 2008, ApJ, 681, 1129

Bongiorno, A., Mignoli, M., Zamorani, G., et al. 2010, A\&A, 510, A56

Bruzual, G., \& Charlot, S. 2003, MNRAS, 344, 1000

Capak, P., Aussel, H., Ajiki, M., et al. 2007, ApJ, 172, 99

Caputi, K. I., Lilly, S. J., Ausse, H., et al. 2008, ApJ, 680, 939

Caputi, K. I., Kovač, K., Bolozonella, M., et al. 2009, ApJ, 691, 91

Chabrier, G. 2003, PASP, 115, 763

Ciotti, L., Ostriker, J. P., \& Proga, D. 2009, ApJ, 699, 89

Colless, M., Dalton, G., Maddox, S., et al. 2001, MNRAS, 328, 1039

Condon, J. J. 1992, ARA\&A, 30, 575

Condon, J. J., Cotton, W. D., Greisen, E. W., et al. 1998, AJ, 115, 1693

Croton, D. J., Springel, V., White, S. D. M., et al. 2006, MNRAS, 365, 11

Cucciati, O., Iovino, A., Kovač, K., et al. 2010, A\&A, submitted

Dressler, A. 1980, ApJ, 236, 351

Giacintucci, S., Venturi, T., Bardelli, S., Dallacasa, D., \& Zucca, E. 2004, A\&A, 419, 71

Hardcastle, M. J., Evans, D. A., \& Croston, J. H. 2007, MNRAS, 376, 1849

Helsdon, S. F., Ponman, T. J., O’Sullivan, E. O., \& Forbes, D. A. 2001, MNRAS, 325,693

Hill, G. J., \& Lilly, S. J. 1991, ApJ, 367, 1

Hopkins, P. F., Robertson, B., Krause, E., Hernquist, L., \& Cox, T. J. 2006, ApJ, 652,107

Ilbert, O., Tresse, L., Zucca, E., et al. 2005, A\&A, 439, 863

Ilbert, O., Capak, P., Salvato, M., et al. 2009, ApJ, 690, 1236

Iovino, A., Cucciati, O., Scodeggio, M., et al. 2010, A\&A, 509, A40

Kauffman, G., Heckman, T. M., Tremonti, C., et al. 2003, MNRAS, 346, 1005

Kauffmann, G., White, S., Heckman, T. M., et al. 2004, MNRAS, 353, 712

Kauffmann, G., Heckman, T. M., \& Best, P. N. 2008, MNRAS, 384, 953

Kewley, L. J., Dopita, M. A., Sutherland, R. S., Heisler, C. A., \& Trevena, J. 2001, ApJ, 556, 121

Koekemoer, A. M., Aussel, H., Calzetti, D., et al. 2007, ApJS, 172, 196

Knobel, C., Lilly, S. J., Iovino, A., et al. 2009, ApJ, 697, 1842

Kovač, K., Lilly, S. J., Cucciati, O., et al. 2010, ApJ, 708, 505

Lacy, M., Storrie-Lombardi, L. J., Sajina, A., et al. 2004, ApJS, 154, 166

Lamareille, F., Mouhcine, M., Contini, T., Lewis, I., \& Maddox, S. 2004, MNRAS, 350, 396

Lamareille, F., Brinchmann, J., Contini, T., et al. 2009, A\&A, 495, 53

Ledlow, M. J., \& Owen, F. 1996, ApJ, 112, 9

Lilly, S. J., Le Fevre, O., Renzini, A., et al. 2007, ApJS, 172, 70

Lilly, S. J., LeBrun, V., Maier, C., et al. 2009, ApJS, 184, 218

Mandelbaum, R., Li, C., Kauffmann, G., \& White, S. D. M. 2009, A\&A, 393, 377

Moustakas, J., Kennicutt, R. C., \& Tremonti, C. A. 2006, ApJ, 624, 755

Marconi, A., \& Hunt, L. K. 2003, ApJ, 589, L21

Miller, N. A., Ledlow, M. J., Owen, F. N., \& Hill, J. M. 2002, AJ, 123, 3018

Mignoli, M., Zamorani, G., Scodeggio, M., et al. 2009, A\&A, 493, 93

Mittal, R., Hudson, D. S., Reiprich, T. H., \& Clarke, T. 2008, A\&A, 501, 835

Pannella, M., Carilli, C. L., Daddi, E., et al. 2009, ApJ, 698, 116

Peng, C. Y., Impey, C. D., Rix, H. W., et al. 2006, ApJ, 649, 616

Pozzetti, L., Bolzonella, M., Lamareille, F., et al. 2007, A\&A, 474, 433

Pozzetti, L., Bolzonella, M., Zamorani, G., et al. 2010, A\&A, submitted [arXiv: 0907.5416]

Salpeter, E. E. 1955, ApJ, 121, 161

Sanders, D. B., Salvato, M., Haussel, H., et al. 2007, ApJS, 172, 86

Scarlata, C., Carollo, C. M., Lilly, S. J., et al. 2007, ApJS, 172, 406

Schinnerer, E., Smolčic, V., Carilli, C. L., et al. 2007, ApJS, 172, 46

Scodeggio, M., Vergani, D., Cucciati, O., et al. 2009, A\&A, 501, 21

Scoville, N., Abraham, R. G., Aussel, H., et al. 2007, ApJS, 172, 1

Shabala, S. S., Ash, S., Alexander, P., \& Riley, J. M. 2008, MNRAS, 388, 625

Silverman, J. D., Kovač, K., Knobel, C., et al. 2009b, ApJ, 695, 171

Silverman, J. D., Lamareille, F., Maier, C., et al. 2009a, ApJ, 696, 396

Smolčic, V. 2009, ApJ, 669, L43

Smolčic, V., Schinnerer, E., Scodeggio, M., et al. 2008, ApJS, 177, 14

Smolčic, V., Zamorani, G., Schinnerer, E., et al. 2009a, ApJ, 696, 24 
Smolčic, V., Schinnerer, E., Zamorani, G., et al. 2009b, ApJ, 690, 610 Vollmer, B., Braine, J., Balkowski, C., Cayatte, V., \& Duschl, W. J. 2001, A\&A, 374,824

Venturi, T., Dallacasa, D., \& Stefanachi, F. 2004, A\&A, 422, 515

Vergani, D., Zamorani, G., Lilly, S. J., et al. 2010, A\&A, 509, A42

Tasca, L., Kneib, J. P., Iovino, A., et al. 2009, A\&A, 503, 379

Zucca, E., Ilbert, O., Bardelli, S., et al. 2006, A\&A, 455, 879

Zucca, E., Bardelli, S., \& Bolzonella, M. 2009, A\&A, 508, 1217

1 INAF - Osservatorio Astronomico di Bologna - via Ranzani 1, 40127 Bologna, Italy e-mail: sandro.bardelli@oabo.inaf.it

2 Max Planck Institut für Astronomie, Königstuhl 17, Heidelberg 69117, Germany

3 California Institute of Technology, MC 105-24, 1200 East California Boulevard, Pasadena, CA 91125, USA

${ }^{4}$ INAF - Osservatorio Astronomico di Arcetri, Largo E. Fermi 5, 50125 Firenze, Italy

5 Institute of Astronomy, Swiss Federal Institute of Technology (ETH Hönggerberg), 8093 Zürich, Switzerland

${ }^{6}$ Space Telescope Science Institute, 3700 San Martin Drive, Baltimore, MD 21218, USA

7 Max-Planck-Institut für extraterrestrische Physik, 84571 Garching, Germany

8 INAF-Istituto di Radioastronomia, via Gobetti 101, 40129 Bologna, Italy

${ }^{9}$ Laboratoire d'Astrophysique de Toulouse-Tarbes, Université de Toulouse, CNRS, 14 avenue Édouard Belin, 31400 Toulouse, France

10 Laboratoire d'Astrophysique de Marseille, Université d'AixMarseille, CNRS, 38 rue Frederic Joliot-Curie, 13388 Marseille, France
11 European Southern Observatory, Karl-Schwarzschild-Strasse 2, Garching 85748, Germany

12 Dipartimento di Astronomia, Università di Padova, Vicolo Osservatorio 5, 35122 Padova, Italy

13 INAF - IASF Milano, via Bassini 15, 20133 Milan, Italy

14 INAF - Osservatorio Astronomico di Brera, via Brera 28, 20121 Milan, Italy

15 INAF - Osservatorio Astronomico di Torino, strada Osservatorio 20, 10025 Pino Torinese, Italy

16 Departement of Astronomy, University of Massachussetts, 710 North Pleasent Street, Amherst, MA 01003, USA

17 Dipartimento di Astronomia, Università di Bologna, via Ranzani 1, 40127 Bologna, Italy

18 LBNL \& BCCP, University of California, Berkeley, CA, 94720, USA

19 Centre de Physique Theorique, Marseille, France

${ }^{20}$ Institut d'Astrophysique de Paris, UMR 7095 CNRS, Université Pierre et Marie Curie, 98 bis Boulevard Arago, 75014 Paris, France

21 Argelander Institut für Astronomie, Auf dem Hügel 71, 53121 Bonn, Germany

22 INAF - Osservatorio di Roma, via di Frascati 33, 00040 Monteporzio Catone (RM), Italy

23 Observatories of the Carnegie Institute of Washington, Santa Barbara Street, Pasadena, CA 91101, USA

${ }^{24}$ Institute for Astronomy, University of Hawaii, 2680 Woodlawn Drive, HI 96822, USA

25 Research Center for Space and Cosmic Evolution, Ehime University, Bunkyo-cho 2-5, Matsuyama 790-8577, Japan

26 Max-Planck-Institut für Astrophysics, 84571 Garching, Germany

27 Instituto de Astrofisica de Andalucia, CSIC, Apdo. 3004, 18080 Granada, Spain

28 Universitats-Sternwarte, Scheinerstrasse 1, 81679 Muenchen, Germany 